Article

\title{
Overdispersed-Poisson Model in Claims Reserving: Closed Tool for One-Year Volatility in GLM Framework
}

\author{
Stefano Cavastracci Strascia ${ }^{*, \dagger}$ and Agostino Tripodi ${ }^{\dagger}$ \\ IVASS, Prudential Supervision, 00187 Rome, Italy; ago.tripodi@gmail.com \\ * Correspondence: stefano.cavastracci@ivass.it; Tel.: +39-6-42133-211 \\ + These authors contributed equally to this work.
}

Received: 14 September 2018; Accepted: 25 November 2018; Published: 5 December 2018

\begin{abstract}
The aim of this paper is to carry out a closed tool to estimate the one-year volatility of the claims reserve, calculated through the generalized linear models (GLM), notably the overdispersedPoisson model. Up to now, this one-year volatility has been estimated through the well-known bootstrap methodology that demands the use of the Monte Carlo method with a re-reserving technique. Nonetheless, this method is time consuming under the calculation point of view; therefore, approximation techniques are often used in practice, such as an emergence pattern based on the link between the one-year volatility — resulting from the Merz-Wüthrich method—and the ultimate volatility—resulting from the Mack method.
\end{abstract}

Keywords: claims reserving; prediction error; claims development result; one year view

\section{Introduction}

About 10 years ago, in Italy, the use of the generalized linear model (GLM) - to estimate the claims reserve began to spread out both in the academic world and in the insurance market; in 2006, some excellent specialized series of lectures on this subject were sponsored by the Concentric Company and held by Richard Verrall of the London Cass Business School, one of the main developers of this implementation-at the stock-exchange offices in Milan. One obstacle, as to the ability to acquire such models in the Italian actuarial practice, was the need to include information about the claim number in this kind of estimate, as we had already done for years with deterministic methodologies.

Today, rather than a higher flexibility in order to better these models inherent predicting ability, a general development of derivative models mostly featuring a different theoretical background and explained in a series of papers issued in later years—can be observed. These models, which have tried to overcome GLM's own limits, can be classified into four categories:

(a) GLMs including families different from exponential class without distribution restrictions GLZ Venter (2007);

(b) Antonio and Beirlant (2008) GLMM (generalized linear mixed model) that allow for overcoming the hypothesis of independence among payments of claims occurring in the same generation but in different years-processed with stochastic simulation techniques;

(c) Bjökwall et al. (2011) GLMs with smoothing effects;

(d) Hudecovà and Pešta (2013) GEE (generalized estimating equations) implementation, where the connection among payments of the same accident year is made through a closed tool.

However, up to now, their use has remained confined to the academic environment for different reasons: GLZ mainly improves the historical data fitting; the GLMMs_-based on a semiparametric 
regression model—could present heavy computational cost in a professional environment; the GLMs with a smoothing effect always pose the risk to distort the information included in companies data; GEEs are possibly among the most interesting ones. Nevertheless, experts who have studied them have formulated neither an exact theory about the starting correlation estimates to be given to the algorithm of optimisation of the parameters input - which is strictly dependent on them-nor an exhaustive definition of the error prediction formulae.

Back to the GLMs, however, it is important to remark that, in 2016, the Casualty Actuarial Society has issued a substantial monograph Taylor and McGuire (2016) about these models, focused on the diagnostics and modification of the regression patterns. This work, on the contrary, is aimed at deriving the volatility of a particular GLM with Over Dispersed Poisson (ODP) distribution through a closed tool, in a one-year horizon framework. This volatility is particularly relevant to calculate the requirement for the reserve risk capital of the internal models, which so far has been calculated through simulation techniques such as the bootstrapping used in two phases-the so called re-reserving. The authors have drawn inspiration from an essay of Merz and Wüthrich (2015), where the volatility estimate for the overall accident years is calculated in the ODP cross-classified model, through the propagation error in physics. This result has been adapted to the GLM model, this way getting a one-year volatility formulae both for each and for the total of the accident years. The evidence we have talked about will be introduced by a description of the GLM application to the loss-reserving problem, with the addition of useful numerical examples.

On a general basis, the claim reimbursements that have not yet been paid at the end of the financial year imply the claims reserve. The nature of such a balance-sheet item estimate is a major risk source for non-life undertakings, due to the problems that its potential underestimation can bring about. In order to get a proper quantification, actuarial methodologies have already been an integral part of the specific estimation process for a long time. This is often due to the lacking of the case by case assessment of each claim file adopted by companies to calculate the ultimate cost representation for the long tail branches. Despite, in comparison with the traditional methods, stochastic methodologies are less ready-to-use, they have several advantages: they are based on explicit and coherent statistical hypothesis; they get the ad hoc adjustments and discretionary estimates to the minimum; beyond the very accurate best estimate of reserve, they provide confidence intervals of the reserve itself in line with fixed probability levels. Notably, through these methodologies, it is always possible to get to an estimate of the first order-moments mean and the second-order moments variance of the reserve distribution. Of course, also the overall probability distribution can be deducted, either through analytical methods - if further appropriate hypothesis are adopted—or through simulation techniques. The use of stochastic methods has been consolidated through the Solvency II project, by reaching a co-ordinated target in a probabilistic key (best estimate added to risk margin) as a prescribed requisite to estimate the claims reserve and the reserve risk capital. Indeed, in such a framework, an exact definition of best estimate, risk margin and reserve risk capital can only be provided by the application of a stochastic model of estimate to the historical time-series of the claims. Here, the risk margin is additional and aimed at clearly quantifying the risk capital yield according to the uncertainty level of the cash flows to come.

In stochastic estimates-beyond the financial kind of uncertainty, linked to the investments yields and to the legal aspects connected with the paying-off delay-three kinds of risk must be taken into account: model risk, estimation risk, and process risk. Model risk means the risk that an unfitting model could be used to represent the phenomenon; the estimation risk is linked to the volatility of the estimator used in order to infer on the model parameters; the process risk is linked to the variance of the phenomenon under scrutiny.

In order to create a connection with the previous practice, many of the stochastic models for the reserving have been built by widening the traditional deterministic techniques, particularly the well-known chain ladder methodology, based on the development of the cumulative payments. Keeping this in mind, we need to emphasise that some of the most used stochastic methods-Mack and 
ODP just allow to make automatic estimates of the reserve and only apply when the basic chain ladder hypothesis are met. The claims reserving working party of 2002 British actuaries has spotted nothing short of 26 qualitative factors to be taken into account in the claims reserving. Nonetheless, this limit is more neglectable in determining the capital requisite because it is function of a volatility quantification. As to the estimate of cash flows of future payments which have already happened, and to estimate the different kinds of risk that have to be taken into account in the risk margin assessment, this work uses the stochastic models evolution included in the GLM class. It is known that such models allow for using different distributions for the response variable and the explicative variable's parameters which are estimated to be linked to the response variable. Therefore, different traditional methods to estimate the claims reserve can be reviewed under this light; as we have already said, claims reserve estimates resulting from particular generalized linear methods indeed match with the ones resulting from deterministic methods to estimate the claims reserve, such as the chain ladder and the separation methods. Some works about the implementation of GLM to estimate the claims reserve are quoted in our bibliography Despeyroux et al. (2003); Englad and Verral (2001); Englad and Verral (2002); Gigante and Sigalotti (2004); Renshaw and Verrall (1998); and Taylor and McGuire (2004). As a general rule, in order to confine the model risk, as a first step, a wide range of models must be considered to pick the one that better fits data, according to a proper good adaptation quantification. In the reference framework - as a measure of goodness of fit to compare the different models-the log-likelihood is estimated in the case of distributions belonging to the exponential family of random variables-for instance gamma, poisson and inverse-Gaussian distribution. The extended quasi-likelihood function introduced by Nelder and Pregibon (1987) is used instead in the semi-parametric case where just the relation between the variance and the mathematical expectation of the response variable is specified.

Once the model has been chosen, the second step consists of approaching data by modifying the regression structure, for example by adding a particular explicative variable to point out an outlier, keeping as a target an optimal figure of the scaled deviance function (over degrees of freedom).

In Section 1, an overview about the use of the generalized linear models to estimate the claims reserve and all the themes connected to it, also with reference to the framework Solvency II, is provided. In Section 2, data organization in the run-off triangle is illustrated, short hints about the widely-used chain ladder method are provided and the notion of claims development result is introduced. In Section 3, the GLM model to estimate the claims reserve is illustrated. In Section 4, the relation to estimate the ultimate volatility through the GLM method is described. Section 5 is the main focus of this paper and explains how to get the algorithm that allows to estimate the one-year volatility through a closed tool in the GLM framework. Finally, in Section 6, a practical case is presented by using an Italian insurance company disguised run-off triangle.

\section{Claims Reserve Estimation}

In non-life undertakings, in order to estimate the claims reserve for accidents still to be paid generated by an insured risk portfolio at the end of the financial year, we generally make reference to the historical payments triangle, updated at the estimate date.

Notably, we assume that the observations concerning payments already made are connected to accidents happened in a limited previous time-framework; thus, sums paid for accidents happened or generated in previous years are available in this kind of diagram.

For each accident year, data are divided into development years, a variable which quantifies the claim payment year.

\subsection{Data Organization}

Given $Y_{i j}$ as the paid sum, with $j$ as the delay in payment for accidents happened in the $i$-th year, usually called incremental payment. These payments are usually represented in the so-called run-off triangle (see Table 1). 
Table 1. Run-off triangle of cumulative payment.

\begin{tabular}{ccccccc}
\hline $\boldsymbol{i} / \boldsymbol{j}$ & $\mathbf{0}$ & $\mathbf{1}$ & $\cdots$ & $j$ & $\cdots$ & $\boldsymbol{J}$ \\
\hline 1 & $Y_{10}$ & $Y_{11}$ & $\cdots$ & $Y_{1 j}$ & $\cdots$ & $Y_{1 J}$ \\
2 & $Y_{20}$ & $Y_{21}$ & $\cdots$ & & & \\
$\vdots$ & $\vdots$ & & & & & \\
$i$ & $Y_{i 0}$ & & $Y_{i j}$ & & & \\
$\vdots$ & $\vdots$ & & & & & \\
$I$ & $Y_{I 0}$ & & & & & \\
\hline
\end{tabular}

Given instead $C_{i, j}=\sum_{k=0}^{j} Y_{i k}$ as the cumulative payment, i.e., the sum paid-off for the $i$ generation within the first $J$ development years, the recursive relation $C_{i, j}=C_{i, j-1}+Y_{i j}$ with $j>0$ is effective. The ratio $F_{i, j-1}=C_{i, j} / C_{i, j-1}$, named link ratio, is the factor connecting the cumulative payment between two close development years-the $j-1$ and the $j$-for the same $i$ generation. Assuming that the payment process of each generation will be surely over within $J$ years, the overall cost of the $i$ generation will be: $C_{i, J}=\sum_{k=0}^{J} Y_{i k}$; writing again the overall cost in the sum of the two addends will make things clearer:

$$
C_{i, J}^{(t)}=\underbrace{\sum_{k=0}^{t-i} Y_{i k}}_{\text {deterministic }}+\underbrace{\sum_{k=t-i+1}^{J} Y_{i k}}_{\text {stochastic }}
$$

since in the $t$ balance-sheet year the first addend is known for sure, while the second is subjected to randomness. Therefore, the claims reserve estimate for the $i$ generation for the $t$ balance-sheet year, concerns the random component of $C_{i, J}^{(t)}$, that is to say, we have: $R_{i}^{(t)}=\sum_{k=t-i+1}^{J} Y_{i k}=C_{t-j, J}^{(t)}-$ $C_{t-j, j}$. We will instead name $\hat{R}_{i}^{(t)}=\sum_{k=t-i+1}^{J} \hat{Y}_{i k}$ the claims reserve estimate, made at the $t$ time, and $\hat{R}^{(t)}=\sum_{i+j>t} \hat{Y}_{i j}$ the overall claims reserve for all generations. In the following passages, we will use $t$ to make reference to the current date of estimate.

\subsection{Chain Ladder Method: Basic Concept}

The idea underlying the chain ladder method is that there is a proportion between the cumulative payments of two close development years, except for an erratic component with a null mean:

$$
C_{i, j+1}^{(t)}=C_{i, j} f_{j}^{(t)}+\epsilon_{i j} \quad i=1, \ldots, I-j-1,
$$

looking at Equation (2), we conclude that, in the chain ladder model, the cumulative payment are showed by a line through the origin for each $j$ development year. If we assume the residuals variance is $\operatorname{Var}\left(\epsilon_{i j}\right)=\sigma^{2} C_{i, j}$, the least square solution for the $f_{j}^{(t)}$ estimate is:

$$
\hat{f}_{j}^{(t)}=\frac{\sum_{k=1}^{t-j-1} C_{k, j+1}}{\sum_{k=1}^{t-j-1} C_{k, j}}=\frac{\sum_{k=1}^{t-j-1} C_{k, j} F_{k, j}}{\sum_{k=1}^{t-j-1} C_{k, j}} j=0,1, \ldots, J-1,
$$

which is the weighted average of all link ratios observed. This approach implies that the cumulative payments $C_{i_{1}, j}$ and $C_{i_{2}, j}$ for $i_{1} \neq i_{2}$ are independent; each ratio $j$, beyond being independent from the $i$ generation, must also have equal first two moments with a fixed $j$, thus the process of claim settlement 
must not have undergone structural changes in time. The ultimate cost $\hat{C}_{i, J}^{(t)}$ estimate is calculated through the use of the factors $\hat{f}_{j}^{(t)}$ :

$$
\hat{C}_{i, J}^{(t)}=C_{i, t-i} \prod_{j=t-i}^{J-1} \hat{f}_{j}^{(t)}
$$

thus the claims reserve estimate is:

$$
\hat{R}_{i}^{(t)}=\hat{C}_{i, J}^{(t)}-C_{i, t-i}
$$

\subsection{The Claims Development Result}

The CDR is the technical result of the evolution of the claim settlement process. In other words, it calculates if the claims reserve $R_{i}^{(t)}$ —set aside in the generic $t$ balance-sheet year, for the $i$ generation-is enough to pay the claims $Y_{i, t-i+1}$, between $t$ and $t+1$ and to set aside the new claims reserve $R_{i}^{(t+1)}$ in $t+1$ formally:

$$
C D R_{i, t+1}=R_{i}^{(t)}-\left(Y_{i, t-i+1}+R_{i}^{(t+1)}\right)=C_{i, J}^{(t)}-C_{i, J}^{(t+1)}
$$

is a random variable if the observation moment is $t$, while it is a deterministic value if the observation moment is $t+1$. In the risk estimate and solvency capital calculation framework, we are interested in $t$ observation random variable, while, in the balance-sheet analysis framework, we are interested in the deterministic aspect observed in $t+1$. Particularly, we have a loss if $C D R_{i, t+1}<0$, while we have a gain with a positive result.

\section{Generalized Linear Models to Estimate the Claims Reserve}

The GLM are a generally wide range of models in which it is possible to define and maximize the likelihood function while estimating the parameters. Assuming that for this function regularity conditions are respected, the parameter estimated through the maximum likelihood function method have got many properties, such as: consistency, asymptotic correctness and asymptotic normality. These properties allow for getting additional information about parameters and calculations about the goodness of fit. Furthermore, the same reserving estimates calculated through the traditional estimation methods can be replicated by using particular kinds of GLM. In our following calculations, with reference to a generic parameter $\alpha$, we will use $\hat{\alpha}$ to make reference to its estimate and the $\tilde{\alpha}$ symbol to make reference to its corresponding estimator.

\subsection{GLM Models Structure}

In GLM models, the response variable is typically represented by observed payments $Y_{i j}$ to estimate the claims reserve, while the accident year and the development year are used for the explicative variables. Notably, the explicative variables are used as qualitative factors and therefore coded through the dummy variables. For GLM models, the following properties are valid:

1. the $Y_{i j}$ are stochastically independent;

2. the density (or probability) function is in exponential family:

$$
f\left(y ; \theta_{i j}, \phi\right)=\exp \left\{\frac{\omega_{i j}}{\phi}\left[y \theta_{i j}-b\left(\theta_{i j}\right)\right]\right\} c\left(y ; \theta_{i j}, \phi\right),
$$

where $\omega_{i j}$ is an indicated weight, $\theta_{i j}$ is the prescribed parameter, $\phi$ is the dispersion parameter independent from $i$, and $j$, and $b($.$) and c($.$) are functions which identify the particular$ exponential family; 
3. the moments can be generalized as follows:

$$
E\left[Y_{i j}\right]=g^{-1}\left(x_{i j}^{\top} \beta\right)=b^{\prime}\left(\theta_{i j}\right) \text { and } \operatorname{Var}\left[Y_{i j}\right]=\frac{\phi}{\omega_{i j}} b^{\prime \prime}\left(\theta_{i j}\right)=\frac{\phi}{\omega_{i j}} V\left(\mu_{i j}\right),
$$

where $x_{i j}$ is the column vector of the explicative variables, $\beta$ is the parameters vector and $g$ is a continuous and invertible function which is called link function. In our following calculations, we will use $\mathrm{h}$ to make reference to the reverse of the link function, i.e., $h=g^{-1}$, while $V\left(\mu_{i j}\right)=b^{\prime \prime}\left(b^{\prime}-1\left(\mu_{i j}\right)\right)$ is the so-called variance function.

We have indicated with $X$ the design matrix, where the generic row is the vector which indicates the explicative variables for the matching response variable calculation, while $\eta=X \beta$ is the linear predictor. Therefore, $g($.$) is the function that links each element of the \eta_{i j}=x_{i j}^{\top} \beta$ linear predictor with $E\left[Y_{i j}\right]=h\left(\eta_{i j}\right)$, i.e., with the mathematical expectation. For the regression parameters vector $\beta=\left(c, a_{1}, \ldots, a_{I}, b_{0}, \ldots, b_{J}\right)^{\top}$, the $c$ parameter indicates a feature in common with all the observations-model intercept-, the $a_{1}, a_{2}, \ldots, a_{I}$ parameters are linked to the accident year, while the $b_{0}, b_{1}, \ldots, b_{J}$ parameters are connected to the payment development year. The model we have created this way will be over-parametrized, and, above all, defined unless an additive constant. To correct this problem, we will assume the link $a_{1}=b_{0}=0$; indeed, such parameters are not included in the $X$ matrix, thus the generic parameter $a_{i}$ and $b_{j}$ indicates the difference from the $c$ intercept.

In GLM models, the $\beta$ parameters estimate is calculated through the maximum likelihood method; this approach allows for calculating the maximum likelihood mathematical expectation estimates given $\hat{\mu}_{i j}=h\left(\eta_{i j}\right)$ with $i+j>t$. The dispersion $\phi$ parameter estimate when it is not known can itself be calculated either through the maximum likelihood method or through consistent estimators-for instance, through the one based on Pearson estimator:

$$
\hat{\phi}=\frac{1}{n-p} \sum_{i+j \geq t} \omega_{i j} \frac{\left(y_{i j}-\hat{\mu}_{i j}\right)^{2}}{V\left(\hat{\mu}_{i j}\right)},
$$

where $n-p$ is the number of the model freedom degrees $n$ is the number of the observed data, $p$ is the number of parameters to be estimated particularly; in this case, we have $n=\frac{I(I+1)}{2}, p=I+J=2 I-1$ and thus $n-p=\frac{I^{2}-3 I+2}{2}$.

Remark 1. The regression structure can be altered by inserting other parameters linked to further explicative variables. We can pick them through preliminary analysis based on data and through inferential analysis to compare models based on adjustment to data validity indicators and on residuals analysis. Notably, a new parameter can be inserted as well, to observe particular interactions between the two variables' accident year and development year, therefore corresponding to particular data.

\subsection{Semi-Parametrical Models}

As we have already said, to define the likelihood function, it is necessary to specify the analytic form of distribution of the response variable, while it is possible to define the quasi-likelihood by specifying only the relation between mean and variance as described by Wedderburn (1974) ${ }^{1}$ :

$$
K(y ; \beta, \phi)=\sum_{i+j \leq t} \omega_{i j} \int_{y_{i j}}^{\mu_{i j}} \frac{y_{i j}-s}{\phi V(s)} d s .
$$

1 Robert W. M. Wedderburn (1947-1975) could have become one of the most distinguished statistics experts of his time due to his early works in this field, but he died at the young age of 28 because of an anaphylactic shock caused by a wasp bite. 
This relation can be used to estimate the $\beta$ parameters. The quasi-likelihood function includes properties that are similar to the likelihood function, therefore also the parameters we can get by maximizing the (10). In the over-dispersed Poisson model with logarithmic link-function instead, the ratio between mean and variance is the following:

$$
E\left[Y_{i j}\right]=\mu_{i j}=e^{c+a_{i}+b_{j}} \quad \text { and } \quad \operatorname{Var}\left[Y_{i j}\right]=\phi V\left(\mu_{i j}\right)=\phi \mu_{i j} .
$$

By inserting Equations (11) into (10), we can get the expression of the quasi-likelihood function for the over-dispersed Poisson model:

$$
K(y ; \beta, \phi)=\sum_{i+j \leq t} \frac{\omega_{i j}}{\phi}\left[y_{i j} \log \frac{\mu_{i j}}{y_{i j}}-\mu_{i j}+y_{i j}\right],
$$

the $\hat{\beta}$ estimate is calculated by searching for the $\beta$ values that maximise Equation (12). The optimization problem can be solved through the Gauss-Newton method.

\subsection{Elements for the Observed Data Goodness of Fit}

One method often used to estimate the observed data model goodness of fit is to analyse the generalized Pearson residuals. Through them, it is possible to analyse the presence of anomalous data or trends. The calculation formula of these residuals is:

$$
r_{i j}=\frac{y_{i j}-\hat{\mu}_{i j}}{\sqrt{V\left(\hat{\mu}_{i j}\right) / \omega_{i j}}} .
$$

Usually, under the hypothesis of residual normality, it may happen that they are included in the critical values \pm 1.96 . In the ODP case, with $\omega_{i j}=1$, the residuals become:

$$
r_{i j}=\frac{y_{i j}-\hat{\mu}_{i j}}{\sqrt{\hat{\phi} \hat{\mu}_{i j}}} .
$$

In order to calculate the overall discrepancy between empirical and theoretical data, as a rule, we use Pearson statistics $\chi^{2}=\sum_{i+j \leq t} \omega_{i j} \frac{\left(y_{i j}-\hat{\mu}_{i j}\right)^{2}}{V\left(\hat{\mu}_{i j}\right)}$ and the deviance:

$$
D(\hat{\mu} ; y)=-2 \sum_{i+j \leq t} \omega_{i j}\left[y_{i j}\left(\hat{\theta}_{i j}-\theta_{i j}^{*}\right)-\left(b\left(\hat{\theta}_{i j}\right)-b\left(\theta_{i j}^{*}\right)\right)\right]
$$

with $\hat{\theta}_{i j}=b^{\prime}-1\left(\hat{\mu}_{i j}\right)$ and $\theta_{i j}^{*}=b^{\prime}-1\left(y_{i j}\right)$. In the quasi-likelihood case, the deviance (15) becomes: $D(\hat{\mu} ; y)=-2 \hat{\phi} K(y ; \beta, \phi)$.

\section{The Claims Reserve Mean Square Error of Prediction}

\subsection{The General Case}

A stochastic model for the claims reserving is a prediction method in which payments to come are modeled through the estimators that are a function of the observed data. Therefore, beyond the variability typical of any random variable-process variance-we also need to take into account the variability inherent in the model parameters estimate-estimation or parameter variance.

First of all, we take into account the random $R$ variable, which indicates the claims reserve. By using a proper model for $R$, we define an $\tilde{R}$ estimator carefully modeled on the observed data and we call mean square error prediction (MSEP)—the following quantity: 


$$
\operatorname{MSEP}(\tilde{R})=E\left[(R-\tilde{R})^{2}\right],
$$

if $\tilde{R}$ is a correct estimator for the $R$ mean-i.e., $E(R)=E(\tilde{R})$ - it will be possible to get the following decomposition:

$$
\begin{aligned}
\operatorname{MSEP}(\tilde{R}) & =E\left[(R-E(R)+E(R)-\tilde{R})^{2}\right] \\
& \approx E\left[(R-E(R))^{2}\right]+E\left[(\tilde{R}-E(R))^{2}\right] \\
& =\underbrace{\operatorname{Var}(R)}_{\text {process }}+\underbrace{\operatorname{Var}(\tilde{R})}_{\text {parameter }} .
\end{aligned}
$$

During Equation (17) differentiation, the covariance term is canceled out because of the hypothesis of independence between past observations and future predictions.

\subsection{GLMs Implementation in Claims Reserving}

In Section 4.1, we have talked about the estimate of historical data distribution parameters. Of course, in order to assess future cash-flows, we need to make predictions and to take into account the prediction errors for the random elements of the lower triangle. To this end, we will assume that the observed data are the result of random variables being stochastically independent and with probability distributions belonging to the same parameters family. This being the hypothesis, on the basis of the estimates and the estimators that we have obtained from run-off data parameters, we can also calculate estimates of distribution and estimators for the random variables of the lower triangle.

Let's assume $\hat{\beta}=\left(\hat{c}, \hat{a}_{1}, \ldots, \hat{a}_{I}, \hat{b}_{0}, \ldots, \hat{b}_{J}\right)^{\top}$ to be the estimate and $\tilde{\beta}=\left(\tilde{c}, \tilde{a}_{1}, \ldots, \tilde{a}_{I}, \tilde{b}_{0}, \ldots, \tilde{b}_{J}\right)^{\top}$ to be the estimator of the maximum likelihood, and $\hat{\phi}$ to be the estimate of the dispersion parameter. For the random variable $Y_{i j}$ with $i+j>t$, we can estimate the mathematical expectation and the variance through:

$$
\begin{gathered}
\hat{E}\left[Y_{i j}\right]=\hat{\mu}_{i j}=h\left(\hat{\eta}_{i j}\right)=h\left(\hat{c}+\hat{a}_{i}+\hat{b}_{j}\right) \\
\text { and } \widehat{\operatorname{Var}}\left[Y_{i j}\right]=\hat{\phi} V\left(\hat{\mu}_{i j}\right) .
\end{gathered}
$$

Let's assume instead $Y_{i j}$ as the transform of the linear predictor $\tilde{Y}_{i j}=h\left(\tilde{\eta}_{i j}\right)=h\left(\tilde{c}+\tilde{a}_{i}+\tilde{b}_{j}\right)$. In the ODP model with a logarithmic link function case, we have:

$$
\begin{aligned}
\hat{E}\left[Y_{i j}\right] & =\hat{\mu}_{i j}=e^{\left(\hat{c}+\hat{a}_{i}+\hat{b}_{j}\right)} \\
\text { and } \widehat{\operatorname{Var}}\left[Y_{i j}\right] & =\hat{\phi} \hat{\mu}_{i j} .
\end{aligned}
$$

We can get the estimate of mathematical expectations of the claims reserve-under the hypothesis of stochastic independence-as sums of the previous ones including the whole group of indexes defining different quantities. We can apply the same to the estimators sums. We have:

$$
\hat{E}\left[R_{i}\right]=\sum_{j=t-i+1}^{J} \hat{\mu}_{i j}, \quad \hat{E}[R]=\sum_{i+j>t} \hat{\mu}_{i j}, \quad \tilde{E}\left[R_{i}\right]=\sum_{j=t-i+1}^{J} \tilde{\mu}_{i j} \quad \text { and } \quad \tilde{E}[R]=\sum_{i+j>t} \tilde{\mu}_{i j} .
$$

Predictions are given by the estimators observed values and they match with the mathematical expectations written above. To calculate the prediction errors instead, we make reference to some asymptotic results about the maximum likelihood estimators of parameters in GLMs. Notably, if $\phi$ is known and if the regularity conditions of the likelihood function are respected, the maximum likelihood estimators satisfy the properties of consistency and asymptotic normality. Therefore, the mathematical expectation of the distribution can be approximated through the $\hat{\beta}$ estimate, while the variance-covariance matrix can be estimated through the inverse of the Fisher information matrix: 


$$
I(\hat{\beta})=-E\left[\left.\frac{\partial^{2} \tilde{l}}{\partial \beta_{h} \partial \beta_{j}}\right|_{\beta=\hat{\beta}}\right],
$$

where $\tilde{l}$ is the r.v. we get by replacing the observations $y_{i j}$-that are the results of the r.v. $Y_{i j}$-in the expression of the log-likelihood function $l$. If $\phi$ is not known, previous results will be valid all the same if we replace with its own consistent estimate; thus, the estimator $\tilde{\beta}=\left(\tilde{c}, \tilde{a}_{1}, \ldots, \tilde{a}_{I}, \tilde{b}_{0}, \ldots, \tilde{b}_{J}\right)^{\top}$ will be generally consistent and asymptotically normal: $\tilde{\beta} \sim N\left(\hat{\beta}, I^{-1}(\hat{\beta})\right)$, where $I^{-1}(\hat{\beta})$ is indeed the estimate of the variance-covariance matrix of the $\tilde{\beta}$ estimator, particularly:

$$
\widehat{\operatorname{Var}}(\hat{\beta})=\left[\begin{array}{cccccc}
\hat{\operatorname{Var}}(\hat{c}) & \ldots & \hat{\operatorname{Cov}}\left(\hat{c}, \hat{a}_{i}\right) & \ldots & \hat{\operatorname{Cov}}\left(\hat{c}, \hat{b}_{j}\right) & \ldots \\
\vdots & & \vdots & & \vdots & \\
\hat{\operatorname{Cov}}\left(\hat{a}_{i}, \hat{c}\right) & \ldots & \hat{\operatorname{Var}}\left(\hat{a}_{i}\right) & \ldots & \hat{\operatorname{Cov}}\left(\hat{a}_{i}, \hat{b}_{j}\right) & \ldots \\
\vdots & & \vdots & & \vdots & \\
\hat{\operatorname{Cov}\left(\hat{b}_{j}, \hat{c}\right)} & \ldots & \hat{\operatorname{Cov}}\left(\hat{b}_{j}, \hat{a}_{i}\right) & \ldots & \hat{\operatorname{Var}}\left(\hat{b}_{j}\right) & \ldots \\
\vdots & & \vdots & & \vdots &
\end{array}\right]=I^{-1}(\hat{\beta}) .
$$

When the mean of a random variable and the one of the estimator match, the MSEP is the sum of the process variance and the parameter variance (see Equation (17)). In the GLM model case, the two predicted values match only in the case of the identity link function, while in the unspecified case we can use the following Taylor first-order approximation:

$$
E\left[\tilde{Y}_{i j}\right]=E\left[h\left(\tilde{\eta}_{i j}\right)\right] \approx h\left(\eta_{i j}\right)+h^{\prime}\left(\eta_{i j}\right) E\left[\tilde{\eta}_{i j}-\eta_{i j}\right]
$$

as the estimator $\tilde{\eta}_{i j}$ is asymptomatically correct, $\tilde{\eta}_{i j} \rightarrow \eta_{i j}$, we can conclude that $E\left[\tilde{Y}_{i j}\right] \approx E\left[Y_{i j}\right]$; therefore, as for the (MSEP), we get:

$$
\operatorname{MSEP}\left(\tilde{Y}_{i j}\right)=\operatorname{Var}\left(Y_{i j}\right)+E\left[\left(\tilde{Y}_{i j}-E\left(Y_{i j}\right)\right)^{2}\right] \approx \operatorname{Var}\left(Y_{i j}\right)+\operatorname{Var}\left(\tilde{Y}_{i j}\right)
$$

with a similar procedure; as for variance, we get:

$$
\operatorname{Var}\left(\tilde{Y}_{i j}\right)=\operatorname{Var}\left[h\left(\tilde{\eta}_{i j}\right)\right] \approx\left[h^{\prime}\left(\hat{\eta}_{i j}\right)\right]^{2} \operatorname{Var}\left(\tilde{\eta}_{i j}\right),
$$

the linear predictor variance estimate $\tilde{\eta}_{i j}$ can be calculated through the variance-covariance matrix (23), and, in particular, we have:

$$
\widehat{\operatorname{Var}}\left(\tilde{\eta}_{i j}\right)=\widehat{\operatorname{Var}}(\tilde{c})+\widehat{\operatorname{Var}}\left(\tilde{a}_{i}\right)+\widehat{\operatorname{Var}}\left(\tilde{b}_{j}\right)+2\left[\widehat{\operatorname{Cov}}\left(\tilde{c}, \tilde{a}_{i}\right)+\widehat{\operatorname{Cov}}\left(\tilde{a}_{i}, \tilde{b}_{j}\right)+\widehat{\operatorname{Cov}}\left(\tilde{c}, \tilde{b}_{j}\right)\right]
$$

or in its compact form:

$$
\widehat{\operatorname{Var}}\left(\tilde{\eta}_{i j}\right)=x_{i j}^{\top} \widehat{\operatorname{Var}}\left(\tilde{\beta}_{i j}\right) x_{i j}
$$

where $x_{i j}$ is the dummy variables vector, variables used to code accident and development year.

Finally, as for the MSEP, we get the following formula:

$$
\widehat{\operatorname{MSE} P}\left(\tilde{Y}_{i j}\right)=\hat{\phi} \hat{\mu}_{i j}+\left[h^{\prime}\left(\hat{\eta}_{i j}\right)\right]^{2} \widehat{\operatorname{Var}}\left(\hat{\eta}_{i j}\right) .
$$

By following a similar procedure, we can calculate the MSEP of the claims reserve for the $i$ accident year, i.e., $\tilde{R}_{i}=\sum_{j=t-i+1}^{J} \tilde{Y}_{i j}$, that, on the basis of Equation (17), is calculated as the process variance $\operatorname{Var}\left(R_{i}\right)$ plus the parameter variance $\operatorname{Var}\left(\tilde{R}_{i}\right)$ sum, i.e.,: 


$$
\operatorname{MSEP}\left(\tilde{R}_{i}\right)=\operatorname{Var}\left(R_{i}\right)+\operatorname{Var}\left(\tilde{R}_{i}\right) .
$$

As for the process variance estimate, we have:

$$
\widehat{\operatorname{Var}}\left(R_{i}\right)=\sum_{j=t-i+1}^{J} \widehat{\operatorname{Var}}\left(Y_{i j}\right)=\hat{\phi} \sum_{j=t-i+1}^{J} \hat{\mu}_{i j},
$$

while as for the parameter variance estimate:

$$
\widehat{\operatorname{Var}}\left(\tilde{R}_{i}\right)=\sum_{j=t-i+1}^{J} \widehat{\operatorname{Var}}\left(\tilde{Y}_{i j}\right)+\sum_{\substack{j_{1}, j_{2}=t-i+1 \\ j_{1} \neq j_{2}}}^{J} \widehat{\operatorname{Cov}}\left(\tilde{Y}_{i, j_{1}}, \tilde{Y}_{i, j_{2}}\right)
$$

where the estimate of the covariances among incremental payments can be calculated in the following way:

$$
\widehat{\operatorname{Cov}}\left(\tilde{Y}_{i, j_{1}}, \tilde{Y}_{i, j_{2}}\right)=h^{\prime}\left(\hat{\eta}_{i, j_{1}}\right) \cdot h^{\prime}\left(\hat{\eta}_{i, j_{2}}\right) \cdot \widehat{\operatorname{Cov}}\left(\tilde{\eta}_{i, j_{1}}, \tilde{\eta}_{i, j_{2}}\right),
$$

while for linear predictor the covariance estimation is:

$$
\widehat{\operatorname{Cov}}\left(\tilde{\eta}_{i, j_{1}}, \tilde{\eta}_{i, j_{2}}\right)=x_{i, j_{1}}^{\top} \widehat{\operatorname{Var}}\left(\tilde{\beta}_{i j}\right) x_{i, j_{2}}
$$

By inserting (26) and (33) in (32), we get:

$$
\widehat{\operatorname{Var}}\left(\tilde{R}_{i}\right)=\sum_{j=t-i+1}^{J}\left[h^{\prime}\left(\hat{\eta}_{i j}\right)\right]^{2} \operatorname{Var}\left(\tilde{\eta}_{i j}\right)+\sum_{\substack{j_{1}, j_{2}=t-i+1 \\ j_{1} \neq j_{2}}}^{J} h^{\prime}\left(\hat{\eta}_{i, j_{1}}\right) \cdot h^{\prime}\left(\hat{\eta}_{i, j_{2}}\right) \cdot \widehat{\operatorname{Cov}}\left(\tilde{\eta}_{i, j_{1}}, \tilde{\eta}_{i, j_{2}}\right),
$$

in the case of the logarithmic link function, Equation (35) can be written in the following way:

$$
\widehat{\operatorname{Var}}\left(\tilde{R}_{i}\right)=\sum_{j=t-i+1}^{J} \hat{\mu}_{i j}^{2} \operatorname{Var}\left(\tilde{\eta}_{i j}\right)+\sum_{\substack{j_{1}, j_{2}=t-i+1 \\ j_{1} \neq j_{2}}}^{J} \hat{\mu}_{i, j_{1}} \hat{\mu}_{i, j_{2}} \widehat{\operatorname{Cov}}\left(\tilde{\eta}_{i, j_{1}}, \tilde{\eta}_{i, j_{2}}\right) .
$$

For the claims reserve total amount, we have instead $\widehat{\operatorname{MSE} P}(\tilde{R})=\widehat{\operatorname{Var}}(R)+\widehat{\operatorname{Var}}(\tilde{R})$ where:

$$
\widehat{\operatorname{Var}}(R)=\sum_{i+j>t} \widehat{\operatorname{Var}}\left(Y_{i j}\right)
$$

and

$$
\widehat{\operatorname{Var}}(\tilde{R})=\sum_{i+j>t} h^{\prime}\left(\hat{\eta}_{i j}\right)^{2} \widehat{\operatorname{Var}}\left(\tilde{\eta}_{i j}\right)+\sum_{\begin{array}{c}
i_{1}+j_{1}>t \\
i_{2}+j_{2}>t \\
\left(i_{1}, j_{1}\right) \neq\left(i_{2}, j_{2}\right)
\end{array}} h^{\prime}\left(\hat{\eta}_{i 1, j_{1}}\right) \cdot h^{\prime}\left(\hat{\eta}_{i 2, j_{2}}\right) \cdot \widehat{\operatorname{Cov}}\left(\tilde{\eta}_{i 1, j_{1}}, \tilde{\eta}_{i 2, j_{2}}\right),
$$

and in the case of the logarithmic link function:

$$
\widehat{\operatorname{Var}}(\tilde{R})=\sum_{i+j>t} \hat{\mu}_{i j}^{2} \widehat{\operatorname{Var}}\left(\tilde{\eta}_{i j}\right)+\sum_{\substack{i_{1}+j_{1}>t \\ i_{2}+j_{2}>t \\\left(i_{1}, j_{1}\right) \neq\left(i_{2}, j_{2}\right)}} \hat{\mu}_{i 1, j_{1}} \hat{\mu}_{i 2, j_{2}} \widehat{\operatorname{Cov}}\left(\tilde{\eta}_{i 1, j_{1}}, \tilde{\eta}_{i 2, j_{2}}\right) .
$$


Finally, for the $\mathrm{ODP}^{2}$ it is possible to re-write the formulae to calculate the MSEP in the following more compact forms:

$$
\begin{aligned}
\widehat{\operatorname{MSE} P}\left(\tilde{R}_{i}\right) & =\hat{\phi} \sum_{j=t-i+1}^{J} \hat{\mu}_{i j} \\
& +\sum_{j=t-i+1}^{J} \hat{\mu}_{i j}^{2} x_{i j}^{\top} \widehat{\operatorname{Var}}(\tilde{\beta}) x_{i j}+\sum_{\substack{j_{1}, j_{2}=t-i+1 \\
j_{1} \neq j_{2}}}^{J} \hat{\mu}_{i j_{1}} \hat{\mu}_{i j_{2}} x_{i_{1}, j_{1}}^{\top} \widehat{\operatorname{Var}}(\tilde{\beta}) x_{i_{2}, j_{2}}
\end{aligned}
$$

and

$$
\begin{aligned}
& \widehat{\operatorname{MSEP}}(\tilde{R})=\hat{\phi} \sum_{i+j>t} \hat{\mu}_{i j} \\
&+\sum_{i+j>t} \hat{\mu}_{i j}^{2} x_{i j}^{\top} \widehat{\operatorname{Var}}(\tilde{\beta}) x_{i j}+\sum_{\substack{i_{1}+j_{1}>t \\
i_{2}+j_{2}>t \\
\left(i_{1}, j_{1}\right) \neq\left(i_{2}, j_{2}\right)}} \hat{\mu}_{i j_{1}} \hat{\mu}_{i j_{2}} x_{i_{1}, j_{1}}^{\top} \widehat{\operatorname{Var}}(\tilde{\beta}) x_{i_{2}, j_{2}} .
\end{aligned}
$$

\section{One-Year Volatility for the Claims Development Results}

\subsection{Overall Accident Years Estimate}

In order to get to a formula that allows for estimating the one-year volatility-i.e., the standard deviation from the CDR random variable as illustrated in Equation (6)-we have used the error propagation technique, as suggested by Röhr (2016), a technique which allows for calculating at the same time the uncertainty both about the parameters estimate and about the random process underlying the data. As we have already described in Section 2.3, the CDR is the technical result of the claims reserve development in a one-year time-framework. Given Equation (6) and the link ratios illustrated in Section 2.2, in the chain ladder framework, the $C D R_{i, t+1}$ is written in the following way:

$$
\widehat{C D R}_{i, t+1}=\hat{C}_{i, J}^{(t)}-\hat{C}_{i, J}^{(t+1)}=\underbrace{C_{i, t-i} \cdot \hat{f}_{t-i}^{(t)}}_{\hat{C}_{i, t-i+1}^{(t)}} \cdot \hat{f}_{t-i+1}^{(t)} \cdot \ldots \cdot \hat{f}_{J-1}^{(t)}-C_{i, t-i+1} \cdot \hat{f}_{t-i+1}^{(t+1)} \cdot \ldots \cdot \hat{f}_{J-1}^{(t+1)} .
$$

It is therefore necessary to estimate the ultimate $\operatorname{cost} \hat{C}_{i, J}^{(t+1)}$ at the $t+1$ time, but, as we have obtained the observations up to the $t$ time, we can use the $\hat{f}_{j}^{(t+1)}$ estimates made on the basis of the observations in $t$. To this purpose, the best approach is to write (42) in the following way:

$$
\widehat{C D R}_{i, t+1}=C_{i, t-i}\left(\prod_{j=t-i}^{J-1} \hat{f}_{j}^{(t)}\right)\left(1-\frac{C_{i, t-i+1} / C_{i, t-i}}{\hat{f}_{t-i}^{(t)}} \prod_{j=t-i+1}^{J-1} \frac{\hat{f}_{j}^{(t+1)}}{\hat{f}_{j}^{(t)}}\right) .
$$

In a priori absence of data, the ratio between two link ratios linked to the same $j$ development year and estimated in a two-years-in-a-raw balance sheet can be calculated through the credibility factor (see Merz and Wüthrich 2015):

$$
\frac{\hat{f}_{j}^{(t+1)}}{\hat{f}_{j}^{(t)}}=\alpha_{j}^{(t)} \frac{C_{t-j, j+1} / C_{t-j, j}}{\hat{f}_{j}^{(t)}}+\left(1-\alpha_{j}^{(t)}\right),
$$

where $\alpha_{j}^{(t)}=\frac{C_{t-j, j}}{\sum_{i=1}^{t-j} C_{i j}}$ is the credibility coefficient.

2 A useful reference about backtesting in relation to the use of the ultimate volatility of predictions is in Leong et al. (2014), winner of the Variance Prize. 
By using (44) for the link ratios, the CDR estimate (43) can be also written in the following way:

$$
C D R_{i, t+1}=\underbrace{\hat{C}_{i, J}^{(t)}}_{\text {ultimate cost in } t}-\underbrace{\hat{C}_{i, J}^{(t)} \frac{C_{i, t-i+1} / C_{i, t-i}}{\hat{f}_{t-i}^{(t)}} \prod_{j=t-i+1}^{J-1}\left(\alpha_{j}^{(t)} \frac{C_{t-j, j+1} / C_{t-j, j}}{\hat{f}_{j}^{(t)}}+\left(1-\alpha_{j}^{(t)}\right)\right)}_{\text {ultimate cost in } t+1, \quad \hat{C}_{i, J}^{(t+1)}} .
$$

As shown by Renshaw and Verrall (1998), between the link ratios, we have calculated through the chain ladder method and the parameters calculated through the GLM method there is the following ratio:

$$
\hat{f}_{j}^{(t)}=1+\frac{e^{\hat{b}_{j+1}}}{\sum_{k=0}^{j} e^{\hat{b}_{k}}}=\frac{\sum_{k=0}^{j+1} e^{\hat{b}_{k}}}{\sum_{k=0}^{j} e^{\hat{b}_{k}}} \quad j=0, \ldots, J-1,
$$

on the (46) basis, as for the link ratios product, we have $\prod_{j=t-i}^{J-1} \hat{f}_{j}^{(t)}=\frac{\sum_{k=0}^{J} e^{\hat{b}_{k}}}{\sum_{k=0}^{t-i} e^{\hat{b}_{k}}}$, thus the ultimate cost estimate can be defined as:

$$
\hat{C}_{t-j, J}^{(t)}=C_{t-j, j} \frac{\sum_{k=0}^{J} e^{\hat{b}_{k}}}{\sum_{k=0}^{j} e^{\hat{b}_{k}}} \quad j=0, \ldots, J-1 .
$$

If we make reference to the cumulative payment $C_{t-j, j+1}$ instead- that in $t$ is a r.v.-we have:

$$
\begin{aligned}
C_{t-j, j+1} & =C_{t-j, j}+Y_{t-j, j+1} \\
& =\hat{C}_{t-j, J}^{(t)} \frac{\sum_{k=0}^{j} e^{\hat{b}_{k}}}{\sum_{k=0}^{J} e^{\hat{b}_{k}}}+Y_{t-j, j+1} \\
& =\hat{C}_{t-j, J}^{(t)} \frac{\sum_{k=0}^{j+1} e^{\hat{b}_{k}}}{\sum_{k=0}^{J} e^{\hat{b}_{k}}}+Y_{t-j, j+1}-\hat{C}_{t-j, J}^{(t)} \frac{e^{\hat{b}_{j+1}}}{\sum_{k=0}^{J} e^{\hat{b}_{k}}} \\
& =\hat{C}_{t-j, J}^{(t)} \frac{\sum_{k=0}^{j+1} e^{\hat{b}_{k}}}{\sum_{k=0}^{J} e^{\hat{b}_{k}}}+Y_{t-j, j+1}-e^{\hat{c}+\hat{a}_{t-j}+\hat{b}_{j+1}}
\end{aligned}
$$

In calculating the last derivative, we have taken into account that $\hat{C}_{t-j, J}^{(t)}=\sum_{k=0}^{J} \hat{Y}_{t-j, k}=$ $e^{\hat{c}+\hat{a}_{t-j}} \sum_{k=0}^{J} e^{\hat{b}_{k}}$. By adding and subtracting the hypothetical value $e^{c+a_{t-j}+b_{j+1}}$ to (48), we get:

$$
C_{t-j, j+1}=\hat{C}_{t-j, J}^{(t)} \frac{\sum_{k=0}^{j+1} e^{\hat{b}_{k}}}{\sum_{k=0}^{J} e^{\hat{b}_{k}}}+\underbrace{Y_{t-j, j+1}-e^{c+a_{t-j}+b_{j+1}}}_{\xi_{t-j, j+1}}+\underbrace{e^{c+a_{t-j}+b_{j+1}}-e^{\hat{c}+\hat{a}_{t-j}+\hat{b}_{j+1}}}_{\zeta_{t-j, j+1}} .
$$

The cumulative paid sums $C_{t-j, j+1}$ of the following year can be broken up-as suggested in Röhr (2016) — through the $\xi_{t-j, j+1}$ residual linked to the process variance and the $\zeta_{t-j, j+1}$ residual linked to the parameter variance.

Keeping in mind (46), (47) and (49), it is possible to get the following ratio:

$$
\begin{aligned}
\frac{C_{t-j, j+1} / C_{t-j, j}}{\hat{f}_{j}^{(t)}} & =\underbrace{\frac{\hat{C}_{t-j, J}^{(t)}}{C_{t-j, j}} \frac{\sum_{k=0}^{j+1} e^{\hat{b}_{k}}}{\sum_{k=0}^{J} e^{\hat{b}_{k}}} \frac{\sum_{k=0}^{j} e^{\hat{b}_{k}}}{\sum_{k=0}^{j+1} e^{\hat{b}_{k}}}}_{=1}+\left(\xi_{t-j, j+1}+\zeta_{t-j, j+1}\right) \frac{1}{C_{t-j, j}} \frac{\sum_{k=0}^{j} e^{\hat{b}_{k}}}{\sum_{k=0}^{j+1} e^{\hat{b}_{k}}} \\
& =1+\left(\xi_{t-j, j+1}+\zeta_{t-j, j+1}\right) \frac{1}{\hat{C}_{t-j, j+1}^{(t)}} .
\end{aligned}
$$


Therefore, the ultimate cost in $(t+1)$ for the $i$ accident year-that is the second addend of Equation (45)—can also be written in the following way:

$$
\hat{C}_{i, J}^{(t+1)}=\hat{C}_{i, J}^{(t)}\left(1+\frac{\xi_{i, t-i+1}+\zeta_{i, t-i+1}}{\hat{C}_{i, t-i+1}^{(t)}}\right) \prod_{j=t-i+1}^{J-1}\left(1+\alpha_{j}^{(t)} \frac{\xi_{t-j, j+1}+\zeta_{t-j, j+1}}{\hat{C}_{t-j, j+1}^{(t)}}\right) .
$$

As a consequence, the cost of the total of all generations, can be calculated by adding the cost of all the accident years:

$$
\sum_{i=t-J+1}^{I} \hat{C}_{i, J}^{(t+1)}=\sum_{i=t-J+1}^{I} \hat{C}_{i, J}^{(t)}\left(1+\frac{\xi_{i, t-i+1}+\zeta_{i, t-i+1}}{\hat{C}_{i, t-i+1}^{(t)}}\right) \prod_{j=t-i+1}^{J-1}\left(1+\alpha_{j}^{(t)} \frac{\xi_{t-j, j+1}+\zeta_{t-j, j+1}}{\hat{C}_{t-j, j+1}^{(t)}}\right) .
$$

Therefore, it is possible to consider the ultimate cost prediction in $t+1$ as a fluctuation of the prediction in the $t$ time, where the $\xi_{t-j, j+1}$ and $\zeta_{t-j, j+1}$ residuals represent the process innovation. A simulation approach would demand the $\xi_{t-j, j+1}$ simulation and the $\zeta_{t-j, j+1}$ estimate through the bootstrap method.

Notice that the two residuals are independent as to the model basic assumptions. To determine a closed tool, we will use a different approach by taking into account Taylor's expansion of such a fluctuation. As Step 1, we consider the fluctuation linked to the process variance, we calculate the derivatives ${ }^{3}$ - making reference to the first residual — and we estimate them in 0 -for every $\xi$ and $\zeta$-thus getting the following weights:

$$
\begin{aligned}
& q_{k+1}^{(t)}=\left.\partial_{\log Y_{t-k, k+1}} \log \left(\sum_{i=t-J+1}^{I} \hat{C}_{i, J}^{(t+1)}\right)\right|_{0} \\
& =\left.\frac{\partial_{\log Y_{t-k, k+1} \sum_{i=t-J+1}^{I} \hat{C}_{i, J}^{(t+1)}}}{\sum_{i=t-J+1}^{I} \hat{C}_{i, J}^{(t+1)}}\right|_{0}=\left.\frac{\partial_{\log Y_{t-k, k+1} \sum_{i=t-k}^{I} \hat{C}_{i, J}^{(t+1)}}}{\sum_{i=t-J+1}^{I} \hat{C}_{i, J}^{(t+1)}}\right|_{0}, \quad t-I \leq k \leq J-1 .
\end{aligned}
$$

To make this calculation easier, we initially consider the first addend of the numerator of Equation (53) derivative development:

$$
\begin{aligned}
& \left.\partial_{\log Y_{t-k, k+1}} \hat{C}_{t-k, J}^{(t+1)}\right|_{0}=\left.\partial_{\log Y_{t-k, k+1}} \hat{C}_{t-k, J}^{(t)}\left(1+\frac{\tilde{\xi}_{t-k, k+1}+\zeta_{t-k, k+1}}{\hat{C}_{t-k, k+1}^{(t)}}\right) \prod_{j=k+1}^{J-1}\left(1+\alpha_{j}^{(t)} \frac{\tilde{\xi}_{t-j, j+1}+\zeta_{t-j, j+1}}{\hat{C}_{t-j, j+1}^{(t)}}\right)\right|_{0} \\
& =\left.\hat{C}_{t-k, J}^{(t)}\left(\partial_{\log Y_{t-k, k+1}} \frac{\xi_{t-k, k+1}}{\hat{C}_{t-k, k+1}^{(t)}}\right) \prod_{j=k+1}^{J-1}\left(1+\alpha_{j}^{(t)} \frac{\xi_{t-j, j+1}+\zeta_{t-j, j+1}}{\hat{C}_{t-j, j+1}^{(t)}}\right)\right|_{0} \\
& =\left.\hat{C}_{t-k, J}^{(t)}\left(\partial_{\log Y_{t-k, k+1}} \frac{Y_{t-k, k+1}}{\hat{C}_{t-k, k+1}^{(t)}}\right) \prod_{j=k+1}^{J-1}\left(1+\alpha_{j}^{(t)} \frac{\xi_{t-j, j+1}^{t}+\zeta_{t-i, j+1}}{\hat{C}_{t-j, j+1}^{(t)}}\right)\right|_{0} \\
& =\left.\hat{C}_{t-k, J}^{(t)} \frac{Y_{t-k, k+1}}{\hat{C}_{t-k, k+1}^{(t)}} \prod_{j=k+1}^{J-1}\left(1+\alpha_{j}^{(t)} \frac{\xi_{t-j, j+1}+\zeta_{t-j, j+1}}{\hat{C}_{t-j, j+1}^{(t)}}\right)\right|_{0} \\
& =\hat{C}_{t-k, J}^{(t)} \frac{e^{c+a_{t-k}+b_{k+1}}}{\hat{C}_{t-k, k+1}^{(t)}} \text { for } t-I \leq k \leq J-2 \text {, }
\end{aligned}
$$

3 The key to understanding how we have calculated these derivatives is that these residuals fluctuate around zero, the first exactly on average and the second only with the condition of a potential bias in the maximum likelihood estimate. Thus, the replacement of the empirical and the estimated figure with the hypothetical unknown value-respectively for $\xi$ and for $\zeta$-is possible. 
and

$$
\begin{aligned}
\left.\partial_{\log Y_{t-J+1, J}} \hat{C}_{t-J+1, J}^{(t+1)}\right|_{0} & =\left.\partial_{\log Y_{t-J+1, J}} \hat{C}_{t-J+1, J}^{(t)}\left(1+\frac{\xi_{t-J+1, J}+\zeta_{t-J+1, J}}{\hat{C}_{t-J+1, J}^{(t)}}\right)\right|_{0} \\
& =\left.\hat{C}_{t-J+1, J}^{(t)}\left(\partial_{\log Y_{t-J+1, J}} \frac{\xi_{t-J+1, J}^{(t)}}{\hat{C}_{t-J+1, J}^{(t)}}\right)\right|_{0} \\
& =\partial_{\left.\log Y_{t-J+1, J} Y_{t-J+1, J}\right|_{0}} \\
& =e^{c+a_{t-J+1}+b_{J}} \quad \text { for } \quad k=J-1 .
\end{aligned}
$$

By applying the same calculation also to the other addends, we get:

$$
q_{k+1}^{(t)}=\frac{\frac{e^{c+a_{t-k}+b_{k+1}}}{\hat{C}_{t-k, k+1}^{(t)}}\left(\hat{C}_{t-k, J}^{(t)}+\alpha_{k}^{(t)} \sum_{i=t-k+1}^{I} \hat{C}_{i, J}^{(t)}\right)}{\sum_{i=t-J+1}^{I} \hat{C}_{i, J}^{(t)}}, \quad t-I \leq k \leq J-1 .
$$

In calculating this derivative, we have kept in mind that $\partial_{\log x} f(x)=x \frac{\partial}{\partial x} f(x)$.

Considering that $\partial_{\log x} \log (f(x))=\frac{x}{f(x)} \frac{\partial}{\partial x} f(x)$ instead, it is possible to write:

$$
\left.\partial Y_{t-k, k+1} \sum_{i=t-J+1}^{I} \hat{C}_{i, J}^{(t+1)}\right|_{0}=\frac{q_{k+1}^{(t)}}{e^{c+a_{t-k}+b_{k+1}}} \sum_{i=t-J+1}^{I} \hat{C}_{i, J}^{(t)} .
$$

As for the $\zeta$ reminder, linked to the parameter error, we initially consider the derivative in relation to the estimate of the intercept parameter $c$ :

$$
\partial_{\hat{c}_{k+1}} \zeta_{t-k, k+1}=\partial_{\hat{c}_{k+1}}\left(e^{c+a_{t-k}+b_{k+1}}-e^{\hat{c}+\hat{a}_{t-k}+\hat{b}_{k+1}}\right)=-e^{\hat{c}+\hat{a}_{t-k}+\hat{b}_{k+1}} .
$$

Thus, deriving the ultimate cost $\sum_{i=t-k}^{I} \hat{C}_{i, J}^{(t+1)}$ with reference to $\hat{c}$, and estimating the derivative in 0 - for each $\xi$ and $\zeta$, for the latter, we also consider the equality for every parameter (component-wise equality) — similarly to what we had already done to calculate Equations (53) and (57), we get:

$$
\left.\partial_{\hat{c}_{k+1}} \sum_{i=t-k}^{I} \hat{C}_{i, J}^{(t+1)}\right|_{0}=-\frac{e^{c+a_{t-k}+b_{k+1}}}{\hat{C}_{t-k, k+1}^{(t)}}\left(\hat{C}_{t-k, J}^{(t)}+\alpha_{j}^{(t)} \sum_{i=t-k+1}^{I} \hat{C}_{i, J}^{(t)}\right)=-q_{k+1}^{(t)} \sum_{i=t-J+1}^{I} \hat{C}_{i, J}^{(t)}
$$

and, in a very similar way, we get the derivative in comparison with the parameters $\hat{a}_{t-k}$ and $\hat{b}_{k+1}$ :

$$
\begin{aligned}
& \left.\partial_{\hat{a}_{t-k} \sum_{i=t-k}^{I} \hat{C}_{i, J}^{(t+1)}}\right|_{0}=-q_{k+1}^{(t)} \sum_{i=t-J+1}^{I} \hat{C}_{i, J}^{(t)}, \\
& \left.\partial_{\hat{b}_{k+1}} \sum_{i=t-k}^{I} \hat{C}_{i, J}^{(t+1)}\right|_{0}=-q_{k+1}^{(t)} \sum_{i=t-J+1}^{I} \hat{C}_{i, J}^{(t)} .
\end{aligned}
$$

By using the above-written derivatives, as to the ratio between the ultimate cost estimated in $t+1$-which in $\mathrm{t}$ is random-and, in $t$, we get to the Taylor's first-order approximation, which is:

$$
\begin{aligned}
& \frac{\sum_{i=t-J+1}^{I} \hat{C}_{i, J}^{(t+1)}}{\sum_{i=t-J+1}^{I} \hat{C}_{i, J}^{(t)}}=\frac{\sum_{i=t-J+1}^{I} \hat{C}_{i, j}^{(t)}\left(1+\frac{\tilde{\xi}_{i, t-i+1}+\bar{\zeta}_{i, t-i+1}}{\hat{c}_{i, t-i+1}^{(t)}}\right) \prod_{j=t-i+1}^{J-1}\left(1+\alpha_{j}^{(t)} \frac{\tilde{\xi}_{t-j, j+1}+\tilde{t}_{t-j, j+1}}{\hat{c}_{t-j, j+1}^{(t)}}\right)}{\sum_{i=t-J+1}^{I} \hat{c}_{i, j}^{(t)}} \\
& \approx 1+\sum_{k=t-I}^{J-1} \frac{q_{k+1}^{(t)}}{e^{c+a_{t-k}+b_{k+1}}} \xi_{t-j, j+1}-\sum_{k=t-I}^{J-1} q_{k+1}^{(t)}\left(c-\hat{c}+a_{t-k}-\hat{a}_{t-k}+b_{k+1}-\hat{b}_{k+1}\right) \\
& =1+\sum_{k=t-I}^{J-1} \frac{q_{k+1}^{(t)}}{\mu_{t-k, k+1}} \xi_{t-j, j+1}-\sum_{k=t-I}^{J-1} q_{k+1}^{(t)}\left(\eta_{t-k, k+1}-\hat{\eta}_{t-k, k+1}\right) \\
& =1+\sum_{k=t-I}^{J-1} \frac{q_{k+1}^{(t)}}{\mu_{t-k, k+1}} \xi_{t-j, j+1}-\sum_{k=t-I}^{J-1} q_{k+1}^{(t)} x_{t-k, k+1}^{\top}(\beta-\hat{\beta}) .
\end{aligned}
$$


Through using Formulae (45) and (61), taking the denominator-after changing its sign-from the first to the second member and by exploiting the assumption of independence among the incremental payments, for the square sum of the CDR, we get to the following first-order approximation:

$$
\begin{aligned}
\left(\sum_{i=t-J+1}^{I} \widehat{C D R}_{i, t+1}\right)^{2} & \approx\left(\sum_{i=t-J+1}^{I} \hat{C}_{i, J}^{(t)}\right)^{2}\left[\sum_{k=t-I}^{J-1} \frac{\left(q_{k+1}^{(t)}\right)^{2}}{\mu_{t-k, k+1}^{2}} \xi_{t-j, j+1}^{2}\right. \\
& \left.+\sum_{k_{1}=t-I}^{J-1} \sum_{k_{2}=t-I}^{J-1} q_{k_{1}+1}^{(t)} q_{k_{2}+1}^{(t)} \cdot x_{t-k, k+1}^{\top}(\beta-\hat{\beta})(\beta-\hat{\beta})^{T} x_{t-k, k+1}\right] \\
& =\left(\sum_{i=t-J+1}^{I} \hat{C}_{i, J}^{(t)}\right)^{2}\left[\sum_{k=t-I}^{J-1} \frac{\left(q_{k+1}^{(t)}\right)^{2}}{\mu_{t-k, k+1}^{2}} \operatorname{Var}\left(Y_{t-k, k+1}\right)\right. \\
& \left.+\sum_{k_{1}=t-I}^{J-1} \sum_{k_{2}=t-I}^{J-1} q_{k_{1}+1}^{(t)} q_{k_{2}+1}^{(t)} \cdot x_{t-k, k+1}^{\top} \operatorname{Var}(\beta) x_{t-k, k+1}\right]
\end{aligned}
$$

In Equation (62), we have omitted the products between the $\xi$ and $\zeta$ residuals because, as they are independent, they will cancel out in Equation (63).

By replacing the parameters estimates with the corresponding unknown values, we can get the MSEP of the the CDR-i.e., of the one-year loss—-for the total of generations:

$$
\begin{aligned}
\widehat{\operatorname{MSE} P}\left(\sum_{i=t-J+1}^{I} \widehat{\mathrm{CDR}} \widehat{\mathrm{R}}_{i, t+1}\right) & =\left(\sum_{i=t-J+1}^{I} \sum_{j=0}^{J} \hat{\mu}_{i j}\right)^{2} \\
& \times[\underbrace{\hat{\phi} \sum_{k=t-I}^{J-1} \frac{\hat{q}_{k+1}^{2}}{\hat{\mu}_{t-k, k+1}}}_{\text {process }}+\underbrace{\hat{q}^{\top} X_{(t+1)} \widehat{\operatorname{Var}}(\hat{\beta}) X_{(t+1)}^{\top}}_{\text {parameter }} \hat{q}],
\end{aligned}
$$

where $X_{(t+1)}$-hat matrix - is the matrix that codes the accident and development years linked to the incremental payments of $t+1$ year through the dummy variables, while $\hat{q}=\left\{\hat{q}_{k+1}\right\}_{k=t-I}^{J-1}$ is the vector of the $q_{k+1}$ weights estimate calculated in (56) and better specified by using the GLM language:

$$
\hat{q}_{k+1}=\frac{\frac{e^{\hat{c}+\hat{a}_{t-k}+\hat{b}_{k+1}}}{\sum_{j=0}^{k+1} e^{\hat{c} \hat{a}_{t-k}+\hat{b}_{j}}}\left(\sum_{j=0}^{J} e^{\hat{c}+\hat{a}_{t-k}+\hat{b}_{j}}+\alpha_{k}^{(t)} \sum_{i=t-k+1}^{I} \sum_{j=0}^{J} e^{\hat{c}+\hat{a}_{i}+\hat{b}_{j}}\right)}{\sum_{i=t-J+1}^{I} \sum_{j=0}^{J} e^{\hat{c}+\hat{a}_{i}+\hat{b}_{j}}} .
$$

Notice that, for $k=0$, we have $t-k+1>I$; under this circumstance, the summation is null. Simplifying, (64) can also be written in an easier way, as explained in (65):

$$
\hat{q}_{k+1}=\frac{\frac{e^{\hat{b}_{k+1}}}{\sum_{j=0}^{k+1} e^{\hat{b}_{j}}}\left(e^{\hat{a}_{t-k}+\alpha_{k}^{(t)} \sum_{i=t-k+1}^{I} e^{\hat{a}_{i}}}\right)}{\sum_{i=t-J+1}^{I} e^{\hat{a}_{i}}} .
$$

\subsection{One-Year Volatility for Accident Year}

In this section, we will illustrate the calculation of the one-year volatility for an $i^{*}$ fixed accident year. Starting from Equation (51), that we write again just for convenience,

$$
\hat{C}_{i^{*}, J}^{(t+1)}=\hat{C}_{i^{*}, J}^{(t)}\left(1+\frac{\xi_{i^{*}, t-i^{*}+1}+\zeta_{i^{*}, t-i^{*}+1}}{\hat{C}_{i^{*}, t-i^{*}+1}^{(t)}}\right) \prod_{j=t-i^{*}+1}^{J-1}\left(1+\alpha_{j}^{(t)} \frac{\xi_{t-j, j+1}+\zeta_{t-j, j+1}}{\hat{C}_{t-j, j+1}^{(t)}}\right)
$$

and we proceed to get an approximation through a Taylor residuals expansion in series. Similarly to what we have done in the previous section, we calculate the partial derivative, at the point $\xi, \zeta=0$, of the ultimate cost estimated in $(t+1)$ : 


$$
s_{i^{*}, k+1}=\left.\partial_{\log X_{t-k, k+1}} \log \left(C_{i^{*}, J}^{(t+1)}\right)\right|_{0}=\left.\frac{\partial_{\log Y_{t-k, k+1} C_{i^{*}, J}^{(t+1)}}}{C_{i^{*}, J}^{(t+1)}}\right|_{0},
$$

by considering first of all the case $i^{*}=t-k$, we get:

$$
\begin{aligned}
& \left.\partial_{\log Y_{t-k, k+1}} C_{i^{*}, J}^{(t+1)}\right|_{0}=\left.\hat{C}_{i^{*}, J}^{(t)} \frac{Y_{i^{*}, t-i^{*}+1}}{\hat{C}_{i^{*}, t-i^{*}+1}^{(t)}} \prod_{j=t-i^{*}+1}^{J-1}\left(1+\alpha_{j}^{(t)} \frac{\xi_{t-j, j+1}+\zeta_{t-j, j+1}}{\hat{C}_{t-j, j+1}^{(t)}}\right)\right|_{0} \\
& =\hat{C}_{i^{*}, J}^{(t)} \underbrace{\frac{e^{c+a_{i^{*}}+b_{t-i^{*}+1}}}{\hat{C}_{i^{*}, t-i^{*}+1}^{(t)}}}_{r_{t-i^{*}+1}^{(t)}}=\hat{C}_{i^{*}, J}^{(t)} r_{t-i^{*}+1}^{(t)} \quad i^{*}=t-k,
\end{aligned}
$$

while, for $i^{*}=2$ :

$$
\begin{aligned}
\partial_{\left.\log \Upsilon_{2, J} C_{2, J}^{(t+1)}\right|_{0}} & =\left.\hat{C}_{2, J}^{(t)} \frac{\gamma_{2, I}}{\hat{C}_{2, J}^{(t)}}\right|_{0} \\
& =e^{c+a_{2}+b_{J}} \\
& =\hat{C}_{2, J}^{(t)} r_{J}^{(t)} \quad i^{*}=2 ;
\end{aligned}
$$

therefore, in the particular case $i^{*}=t-k$, we have that $s_{t-i^{*}+1}^{(t)}=r_{t-i^{*}+1^{\prime}}^{(t)}$, while for $i^{*}>t-k$ the logarithmic (67) becomes:

$$
\begin{aligned}
& \left.\partial_{\log Y_{t-k, k+1}} C_{i^{*}, J}^{(t+1)}\right|_{0}=\left.\hat{C}_{i^{*}, J}^{(t)}\left(1+\frac{\tilde{\xi}_{i t-i^{*}+1}+\bar{\zeta}_{i t-i^{*}+1}}{\hat{C}_{i t-i^{*}+1}^{(t)}}\right) \alpha_{k}^{(t)} \frac{Y_{t-k, k+1}}{\hat{C}_{t-k, k+1}^{(t)}} \prod_{\substack{j=t-i^{*}+1 \\
j \neq k}}^{J-1}\left(1+\alpha_{j}^{(t)} \frac{\tilde{\xi}_{t-j, j+1}+\zeta_{t-i, j+1}}{\hat{C}_{t-j, j+1}^{(t)}}\right)\right|_{0} \\
& =\hat{C}_{i^{*}, J}^{(t)} \alpha_{k}^{(t)} \frac{e^{c+a_{t-k}+b_{k+1}}}{\hat{C}_{t-k, k+1}^{(t)}}=\hat{C}_{i^{*}, J}^{(t)} \underbrace{\alpha_{k}^{(t)} r_{k+1}^{(t)}}_{s_{i^{*}, k+1}^{(t)}} i^{*}>t-k
\end{aligned}
$$

and we have $s_{i^{*}, k+1}^{(t)}=\alpha_{k}^{(t)} r_{k+1}^{(t)}$. In the particular case of the GLM estimate model, with logarithmic link function and ODP distribution, we get the following simplified form for the $r_{k+1}^{(t)}$ ratio:

$$
r_{k+1}^{(t)}=\frac{e^{b_{k+1}}}{\sum_{j=0}^{k+1} e^{b_{j}}}=1-\frac{1}{f_{k}^{(t)}} \quad k=0, \ldots, J-1,
$$

similarly to (57), keeping in mind that $\partial_{\log x} \log (f(x))=\frac{x}{f(x)} \frac{\partial}{\partial x} f(x)$, we get:

$$
\left.\partial_{Y_{t-k, k+1}} \hat{C}_{i^{*}, J}^{(t+1)}\right|_{0}=\left\{\begin{array}{cc}
\frac{\hat{C}_{i^{*}, I_{t-i^{*}+1}^{(t)}}^{(t)}}{e^{c+a_{i *}^{*}+b_{t-i^{*}+1}},} & i^{*}=t-k, \\
\frac{\hat{C}_{i^{*}, J}^{(t)} J_{k}^{(t)} r_{k+1}^{(t)}}{e^{c+a_{t-k}+b_{k+1}},} & i^{*}>t-k,
\end{array}\right.
$$

equally, by taking the partial derivatives of the $\zeta$ residuals in comparison with the GLM model parameters written in Equations (58) and (59), we get the partial derivatives—at the $\xi, \zeta=0$ point-of the ultimate cost $\hat{C}_{i^{*}, J}^{(t+1)}$, for example in comparison with $\hat{b}_{k+1}$ :

$$
\left.\partial_{\hat{b}_{k+1}} \hat{C}_{i^{*}, J}^{(t+1)}\right|_{0}=\left\{\begin{array}{lll}
-\hat{C}_{i^{*},}^{(t)} \frac{e^{c+a_{i^{*}}+b_{t-i^{*}+1}}}{\hat{C}_{i^{*}, t-i^{*}+1}}, & =-\hat{C}_{i^{*}, J}^{(t)} r_{t-i^{*}+1}^{(t)} & i^{*}=t-k, \\
-\hat{C}_{i^{*}, J}^{(t)} \frac{e^{c+a_{t-k}+b_{k+1}}}{\hat{C}_{t-k, k+1}^{(t)}} \alpha_{k}^{(t)},= & -\hat{C}_{i^{*}, J}^{(t)} \alpha_{k}^{(t)} r_{k+1}^{(t)} & i^{*}>t-k .
\end{array}\right.
$$


By using previous results, for the $i^{*}$ generation, we get the following Taylor approximation for the ratio between the ultimate cost estimate done in $t+1$ and the one done in $t$ :

$$
\begin{aligned}
\frac{\hat{C}_{i^{\prime}, J}^{(t+1)}}{\hat{C}_{i^{*}, J}^{(t)}} & \approx 1+\sum_{k=t-i^{*}}^{J-1} \frac{s_{i^{*}, k+1}^{(t)}}{\mu_{t-k, k+1}} \xi_{t-k, k+1}-\sum_{k=t-i^{*}}^{J-1} s_{i^{*}, k+1}^{(t)}\left(\eta_{t-k, k+1}-\hat{\eta}_{t-k, k+1}\right) \\
& =1+\sum_{k=t-i^{*}}^{J-1} \frac{s_{i^{*}, k+1}^{(t)}}{\mu_{t-k, k+1}} \xi_{t-k, k+1}-\sum_{k=t-i^{*}}^{J-1} s_{i^{*}, k+1}^{(t)} x_{t-k, k+1}^{\top}(\beta-\hat{\beta}) .
\end{aligned}
$$

Therefore, in a way very similar to Equation (62), for the square claims' development ratio, we get the following Taylor's first-order expansion:

$$
\begin{aligned}
\widehat{C D R_{i^{*}, t+1}} & \approx\left(\hat{C}_{i^{*}, J}^{(t)}\right)^{2}\left\{\sum_{k=t-i^{*}}^{J-1}\left(\frac{s_{i^{*}, k+1}^{(t)}}{\mu_{t-k, k+1}} \xi_{t-k, k+1}\right)^{2}\right. \\
& \left.+\sum_{k_{1}=t-i^{*}}^{J-1} \sum_{k_{2}=t-i^{*}}^{J-1} s_{i^{*}, k_{1}+1}^{(t)} s_{i^{*}, k_{2}+1}^{(t)} x_{t-k_{1}, k_{1}+1}^{\top}(\beta-\hat{\beta})(\beta-\hat{\beta})^{\top} x_{t-k_{2}, k_{2}+1}\right\} \\
& =\left(\hat{C}_{i^{*}, J}^{(t)}\right)^{2}\left\{\sum_{k=t-i^{*}}^{J-1}\left(\frac{s_{i^{*}, k+1}^{(t)}}{\mu_{t-k, k+1}}\right)^{2} \operatorname{Var}\left(Y_{t-k, k+1}\right)\right. \\
& \left.+\sum_{k_{1}=t-i^{*}}^{J-1} \sum_{k_{2}=t-i^{*}}^{J-1} s_{i^{*}, k_{1}+1^{*}}^{(t)} s_{i^{*}, k_{2}+1}^{(t)} x_{t-k_{1}, k_{1}+1}^{\top} \operatorname{Var}(\beta) x_{t-k_{2}, k_{2}+1}\right\} .
\end{aligned}
$$

Finally, as for the MSEP estimate, we have the following expression:

$$
\begin{aligned}
& \widehat{\operatorname{MSE} P}\left(\widehat{C D R}_{i^{*}, t+1}\right)=\left(\sum_{j=0}^{J} e^{\hat{\imath}+\hat{a}_{i^{*}}+\hat{b}_{j}}\right)^{2} \\
& \times \quad\left(\hat{\phi} \sum_{k=t-i^{*}}^{J-1} \frac{\hat{s}_{i^{*}, k+1}^{2}}{\hat{\mu}_{t-k, k+1}}+\hat{s}_{\left(i^{*}\right)}^{\top} X_{i^{*},(t+1)} \widehat{\operatorname{Var}}(\hat{\beta}) X_{i^{*},(t+1)}^{\top} \hat{\mathcal{s}}_{\left(i^{*}\right)}\right),
\end{aligned}
$$

where $\hat{s}_{\left(i^{*}\right)}$ is a length $i^{*}-1$ vector whose elements are:

$$
\hat{s}_{i^{*}, k+1}=\left\{\begin{array}{ll}
\hat{r}_{t-i^{*}+1} & t-k=i^{*} \\
\hat{r}_{k+1} \alpha_{k}^{(t)} & 2 \leq t-k<i^{*}
\end{array} \quad k=t-i^{*}, \ldots, J-1,\right.
$$

while $X_{i^{*},(t+1)}$ is the projection matrix - hat matrix — that encodes the incremental payments of the $t+1$ following year for the $i^{*}$ generations and the previous ones.

\section{Numerical Investigation}

In this section, we introduce a numerical application which compares the closed tool method (CT) -illustrated in this paper-with the well-known bootstrapping (BS) method-with re-reserving-whose results are found through Monte Carlo simulation. The triangle used for the comparison is the one represented in Table 2; they are the third party liability segment payments of an Italian company, for obvious privacy reasons, data have been disguised. In Table 3, we have copied the parameters and their standard error estimate calculated by applying the quasi-likelihood (12), while applying (9), we get $\hat{\phi}=410.8964$. In Tables 4 and 5, there are the $\hat{\alpha}_{k}, \hat{q}_{k+1}, \hat{\mu}_{t-k, k+1}, \hat{r}_{k+1}$ and $\hat{s}_{i^{*}}$ values resulting from the GLM model and it is necessary to apply Equations (63) and (76) formulae linked to the MSEP and CDR one-year closed tool estimate that is written on the CT column of Table 6. As we can see from results of Table 6, the proposed formulae to estimate the MSEP produce results similar to the ones calculated with simulation techniques-re-reserving-, bearing in mind that these results include the simulation error.

The ratio between one year and ultimate volatility makes evident the long tail nature of this general liability's triangle. With regard to the new estimated parameters $\alpha^{\prime} s, q^{\prime} s, r^{\prime}$ s and $s^{\prime}$ s, we can provide some interpretations: 
- the coefficients of credibility alpha's quantify the weight, in terms of influence, of the accident year in the next development factor calculation (decreasing for the more recent accident year);

- the $q$ 's indicate the contribution to the overall volatility from the first development year to the latest development year and form a typical $u$-shape due to the level of the payment in the first year and to the small uncertainty for the oldest years for which residual payments relative to the still open claims over the ultimate cost are low;

- the $r^{\prime}$ s stand for the weight of the $k$-th development year parameter above the first $k$ parameters decreasing with the increase of development year;

- the $s^{\prime}$ s involved directly in the accident year volatility is a function based on $r$ interesting the next year development for this accident year plus the subsequent development year with credibility decreasing coefficients.

All of the results shown in this section are obtained by using $\mathrm{R}$ software; the code is reported in vignettes 1,2 and 3. The package ChainLadder is requested and must be previously installed because it is not included in the default configuration. In order to replicate results in Table 3, Code 1 has to be run after the run-off triangle showed in Table 2 is uploaded and named Incremetal.Paid. Code 2 computes the outcome of Table 7 and the R Code 3 gives as outcome Tables 4-6.

Table 2. Incremental payment used in the empirical application $(, 000)$.

\begin{tabular}{|c|c|c|c|c|c|c|c|c|c|c|c|c|c|}
\hline$i / j$ & 0 & 1 & 2 & 3 & 4 & 5 & 6 & 7 & 8 & 9 & 10 & 11 & 12 \\
\hline 2 & 22,382 & 41,502 & 26,508 & 19,734 & 18,715 & 13,983 & 12,885 & 16,371 & 7921 & 7204 & 4428 & 12,897 & \\
\hline 4 & 26,830 & 52,347 & 37,324 & 23,590 & 18,248 & 13,895 & 13,142 & 11,119 & 9429 & 5057 & & & \\
\hline 5 & 26,868 & 62,313 & 33,772 & 22,925 & 16,341 & 12,419 & 12,646 & 9459 & 6658 & & & & \\
\hline 6 & 28,470 & 56,097 & 41,672 & 24,843 & 22,818 & 18,787 & 16,947 & 14,942 & & & & & \\
\hline 8 & 24,101 & 58,520 & 38,749 & 22,449 & 16,008 & 12,506 & & & & & & & \\
\hline 9 & 22,714 & 48,707 & 28,970 & 18,798 & 13,369 & & & & & & & & \\
\hline 10 & 19,973 & 38,262 & 23,298 & 14,819 & & & & & & & & & \\
\hline 11 & 17,252 & 36,994 & 24,361 & & & & & & & & & & \\
\hline 12 & 17,591 & 30,074 & & & & & & & & & & & \\
\hline 13 & 16,907 & & & & & & & & & & & & \\
\hline
\end{tabular}

Table 3. Estimation of GLM parameter using the data in Table 2.

\begin{tabular}{cccccc}
\hline Parameter & Estimate & Std. Error & Parameter & Estimate & Std. Error \\
\hline$\hat{c}$ & 10.1263 & 0.0572 & & & \\
$\hat{a}_{2}$ & -0.0883 & 0.0620 & $\hat{b}_{1}$ & 0.7024 & 0.0468 \\
$\hat{a}_{3}$ & -0.0715 & 0.0629 & $\hat{b}_{2}$ & 0.3132 & 0.0513 \\
$\hat{a}_{4}$ & 0.0155 & 0.0620 & $\hat{b}_{3}$ & -0.0972 & 0.0579 \\
$\hat{a}_{5}$ & 0.0126 & 0.0628 & $\hat{b}_{4}$ & -0.3241 & 0.0635 \\
$\hat{a}_{6}$ & 0.1579 & 0.0614 & $\hat{b}_{5}$ & -0.5254 & 0.0703 \\
$\hat{a}_{7}$ & 0.1551 & 0.0627 & $\hat{b}_{6}$ & -0.5737 & 0.0753 \\
$\hat{a}_{8}$ & 0.0425 & 0.0662 & $\hat{b}_{7}$ & -0.6904 & 0.0843 \\
$\hat{a}_{9}$ & -0.1261 & 0.0716 & $\hat{b}_{8}$ & -1.0112 & 0.1051 \\
$\hat{a}_{10}$ & -0.3171 & 0.0795 & $\hat{b}_{9}$ & -1.2910 & 0.1317 \\
$\hat{a}_{11}$ & -0.3326 & 0.0858 & $\hat{b}_{10}$ & -1.4622 & 0.1643 \\
$\hat{a}_{12}$ & -0.4592 & 0.1044 & $\hat{b}_{11}$ & --0.9285 & 0.1553 \\
$\hat{a}_{13}$ & -0.3909 & 0.1660 & $\hat{b}_{12}$ & -0.2665 & 0.1573 \\
\hline
\end{tabular}


Listing 1. Code for Table 3: Estimation GLM parameters.

Incremetal.Paid \# Triangle of incremental paid in Table 2, it must be imported in $R$

claims <- as.vector(Incremetal.Paid) \# Incremental paid as vector

n.origin <- nrow(Incremetal.Paid)

n.dev <- ncol (Incremetal.Paid)

origin <- factor(row <- rep(1:n.origin, n.dev)) \# accident year as vector

dev <- factor(col <- rep(1:n.dev, each=n.origin)) \# development year as vector

W <- data.frame(claims=claims, origin=origin, dev=dev)

model <- glm(claims origin + dev, family = quasipoisson(), subset=!is.na(claims),

data $=W$ )

Model.Summary <- summary(model) \# summary of glm model

Table.3 <- Model.Summary\$coefficients[,c('Estimate','Std. Error')]

rownames (Table.3)<- c('c',paste('a',2:n.origin, sep=" "), paste('b',1:(n.origin-1), sep=""))

colnames (Table.3) <- c('Estimate', 'Std. Error')

Table. 3

Table 4. Estimation of $\alpha, q$ and $\mu$.

\begin{tabular}{cccccc}
\hline $\boldsymbol{k}$ & $\mathbf{1 3 - k}$ & $\hat{\boldsymbol{\alpha}}_{\boldsymbol{k}}^{(\mathbf{1 3})}$ & $\hat{\boldsymbol{q}}_{\boldsymbol{k}+\mathbf{1}}$ & $\hat{\boldsymbol{\mu}}_{\boldsymbol{t}-\boldsymbol{k}, \boldsymbol{k}+\mathbf{1}}$ & $\hat{\boldsymbol{r}}_{\boldsymbol{k}+\mathbf{1}}^{(\mathbf{1 3})}$ \\
\hline 0 & 13 & 0.0569 & 0.0415 & 34127.94 & 0.6687 \\
1 & 12 & 0.0563 & 0.0192 & 21598.78 & 0.3118 \\
2 & 11 & 0.0677 & 0.0127 & 16260.70 & 0.1714 \\
3 & 10 & 0.0738 & 0.0097 & 13162.94 & 0.1202 \\
4 & 9 & 0.0965 & 0.0094 & 13026.95 & 0.0895 \\
5 & 8 & 0.1264 & 0.0108 & 14693.99 & 0.0786 \\
6 & 7 & 0.1619 & 0.0115 & 14633.21 & 0.0653 \\
7 & 6 & 0.1937 & 0.0096 & 10647.17 & 0.0453 \\
8 & 5 & 0.2077 & 0.0075 & 6959.96 & 0.0331 \\
9 & 4 & 0.2630 & 0.0078 & 5882.08 & 0.0271 \\
10 & 3 & 0.3271 & 0.0158 & 9194.30 & 0.0442 \\
11 & 2 & 0.4779 & 0.0412 & 17527.56 & 0.0789 \\
\hline
\end{tabular}

Table 5. Estimation $s_{\left(i^{*}\right)}$.

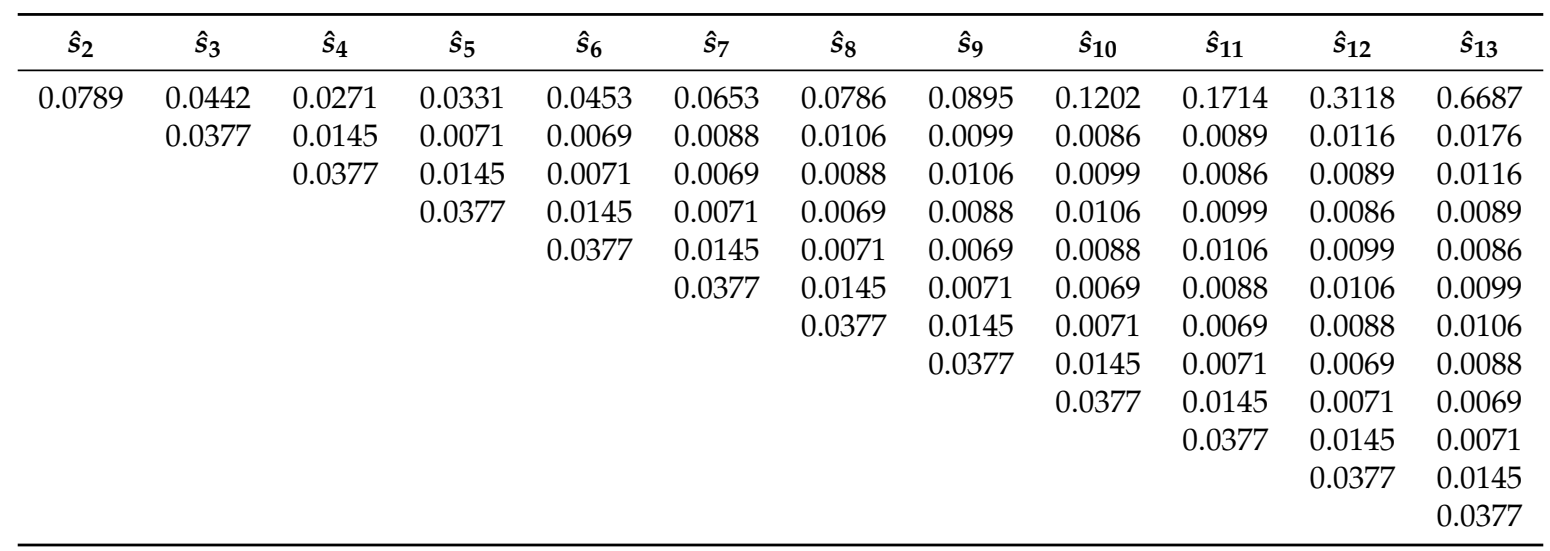


Listing 2. Code for Table 7: Estimation rMSEP ultimate.

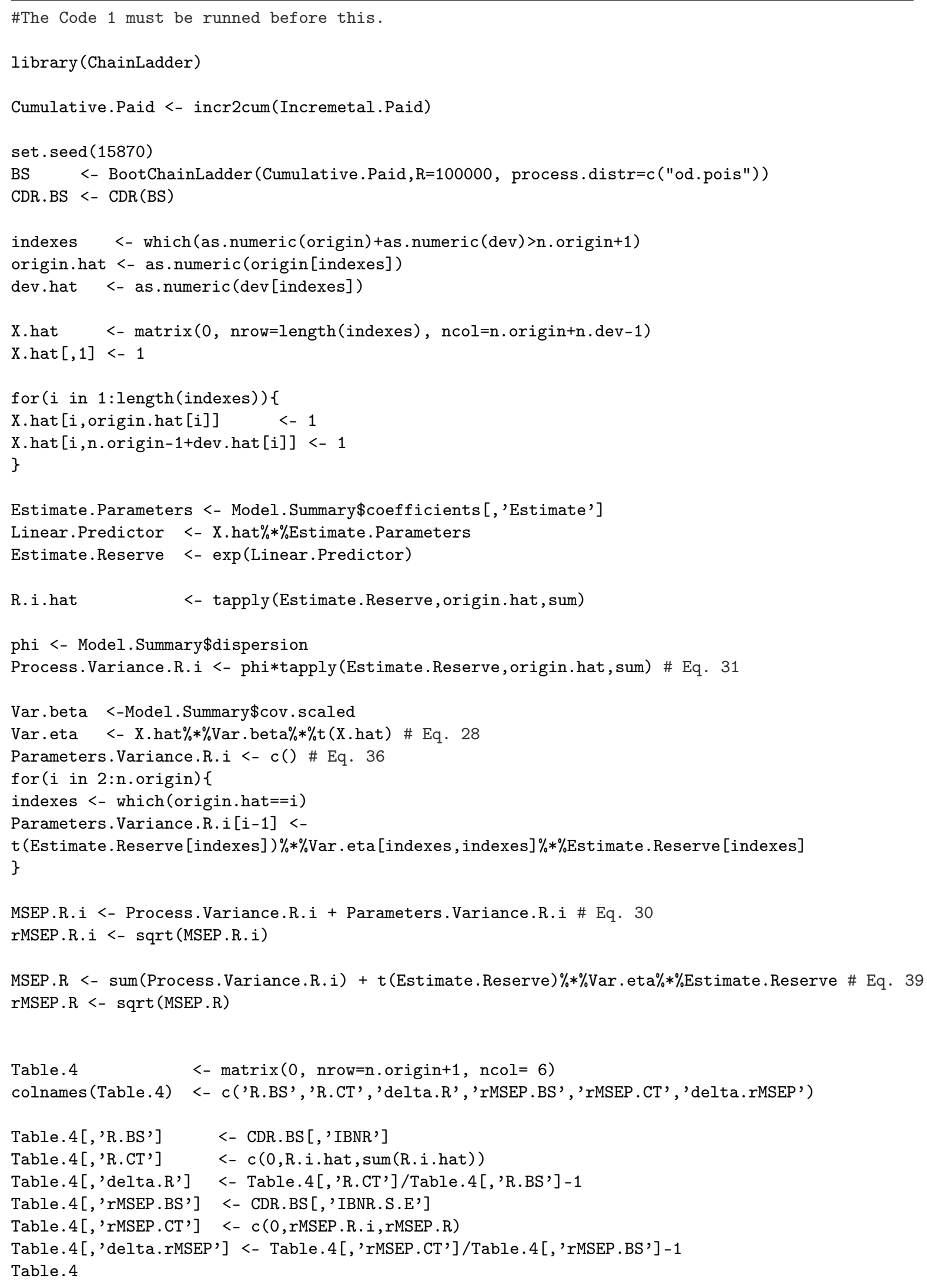


Table 6. Estimation of rMSEP one-year: bootstrapping vs. closed tool.

\begin{tabular}{|c|c|c|c|c|c|c|c|c|}
\hline \multirow{2}{*}{ a.y. } & \multicolumn{2}{|c|}{$r \widehat{M S E P}\left(C D R_{i^{*}, t+1}\right)$} & \multirow{2}{*}{$\Delta \%$} & \multicolumn{2}{|c|}{$\sigma \%$} & \multirow{2}{*}{$\Delta$} & \multicolumn{2}{|c|}{$\frac{r \widehat{M S E P}\left(C D R_{i^{*}, t+1}\right)}{r \widehat{M S E P}\left(\widehat{R}_{i}\right)}$} \\
\hline & BS & $\mathrm{CT}$ & & BS & $\mathrm{CT}$ & & BS & $\mathrm{CT}$ \\
\hline 1 & 0 & 0 & - & - & - & - & - & - \\
\hline 2 & 3888 & 3870 & $-0.46 \%$ & $22.12 \%$ & $22.08 \%$ & $-0.04 \%$ & $100.00 \%$ & $100.00 \%$ \\
\hline 3 & 3238 & 3234 & $-0.12 \%$ & $11.96 \%$ & $11.97 \%$ & $0.01 \%$ & $68.54 \%$ & $68.52 \%$ \\
\hline 4 & 3083 & 3073 & $-0.32 \%$ & $8.70 \%$ & $8.69 \%$ & $-0.01 \%$ & $56.59 \%$ & $56.47 \%$ \\
\hline 5 & 3242 & 3233 & $-0.28 \%$ & $7.67 \%$ & $7.66 \%$ & $-0.01 \%$ & $54.97 \%$ & $54.98 \%$ \\
\hline 6 & 3980 & 3969 & $-0.28 \%$ & $6.68 \%$ & $6.67 \%$ & $-0.01 \%$ & $55.91 \%$ & $55.72 \%$ \\
\hline 7 & 4477 & 4473 & $-0.09 \%$ & $6.05 \%$ & $6.05 \%$ & $0.00 \%$ & $56.53 \%$ & $56.43 \%$ \\
\hline 8 & 4494 & 4490 & $-0.09 \%$ & $5.56 \%$ & $5.56 \%$ & $0.00 \%$ & $54.46 \%$ & $54.53 \%$ \\
\hline 9 & 4319 & 4333 & $0.32 \%$ & $5.31 \%$ & $5.33 \%$ & $0.02 \%$ & $52.08 \%$ & $52.24 \%$ \\
\hline 10 & 4535 & 4538 & $0.07 \%$ & $5.64 \%$ & $5.65 \%$ & $0.01 \%$ & $53.53 \%$ & $53.50 \%$ \\
\hline 11 & 5705 & 5691 & $-0.25 \%$ & $5.98 \%$ & $5.97 \%$ & $-0.01 \%$ & $57.11 \%$ & $56.98 \%$ \\
\hline 12 & 8364 & 8341 & $-0.27 \%$ & $7.91 \%$ & $7.90 \%$ & $-0.01 \%$ & $67.22 \%$ & $67.34 \%$ \\
\hline 13 & 21,651 & 21,616 & $-0.16 \%$ & $14.69 \%$ & $14.69 \%$ & $0.00 \%$ & $86.09 \%$ & $86.17 \%$ \\
\hline Tot & 38,603 & 38,578 & $-0.06 \%$ & $4.56 \%$ & $4.56 \%$ & $0.00 \%$ & $73.09 \%$ & $73.18 \%$ \\
\hline
\end{tabular}

Table 7. Estimation of rMSEP ultimate: bootstrapping vs. closed tool.

\begin{tabular}{ccccccc}
\hline & \multicolumn{3}{c}{$\hat{\boldsymbol{R}}_{\boldsymbol{i}}$} & & \multirow{2}{*}{$\boldsymbol{\Delta} \%$} & \multicolumn{2}{c}{$\boldsymbol{r} \widehat{\mathbf{M S E P}}\left(\hat{\boldsymbol{R}}_{\boldsymbol{i}}\right)$} & \multirow{2}{*}{$\boldsymbol{\Delta} \%$} \\
\cline { 2 - 3 } & $\mathbf{B S}$ & $\mathbf{C T}$ & & $\mathbf{B S}$ & $\mathbf{C T}$ & \\
\hline 1 & 0 & 0 & 0 & 0 & - & - \\
2 & 17,573 & 17,528 & $-0.26 \%$ & 3888 & 3870 & $-0.46 \%$ \\
3 & 27,068 & 27,018 & $-0.18 \%$ & 4724 & 4720 & $-0.08 \%$ \\
4 & 35,429 & 35,356 & $-0.21 \%$ & 5448 & 5442 & $-0.11 \%$ \\
5 & 42,295 & 42,212 & $-0.20 \%$ & 5898 & 5880 & $-0.31 \%$ \\
6 & 59,560 & 59,463 & $-0.16 \%$ & 7118 & 7123 & $0.07 \%$ \\
7 & 74,021 & 73,930 & $-0.12 \%$ & 7920 & 7926 & $0.08 \%$ \\
8 & 80,879 & 80,752 & $-0.16 \%$ & 8252 & 8234 & $-0.22 \%$ \\
9 & 81,354 & 81,245 & $-0.13 \%$ & 8293 & 8295 & $0.02 \%$ \\
10 & 80,401 & 80,285 & $-0.14 \%$ & 8472 & 8483 & $0.13 \%$ \\
11 & 95,412 & 95,309 & $-0.11 \%$ & 9989 & 9988 & $-0.01 \%$ \\
12 & 105,715 & 105,579 & $-0.13 \%$ & 12,443 & 12,386 & $-0.46 \%$ \\
13 & 147,336 & 147,172 & $-0.11 \%$ & 25,149 & 25,085 & $-0.25 \%$ \\
Tot & 847,041 & 845,851 & $-0.14 \%$ & 52,813 & 52,714 & $-0.19 \%$ \\
\hline
\end{tabular}

Listing 3. Code for Tables 4-6: Estimation rMSEP one-year.

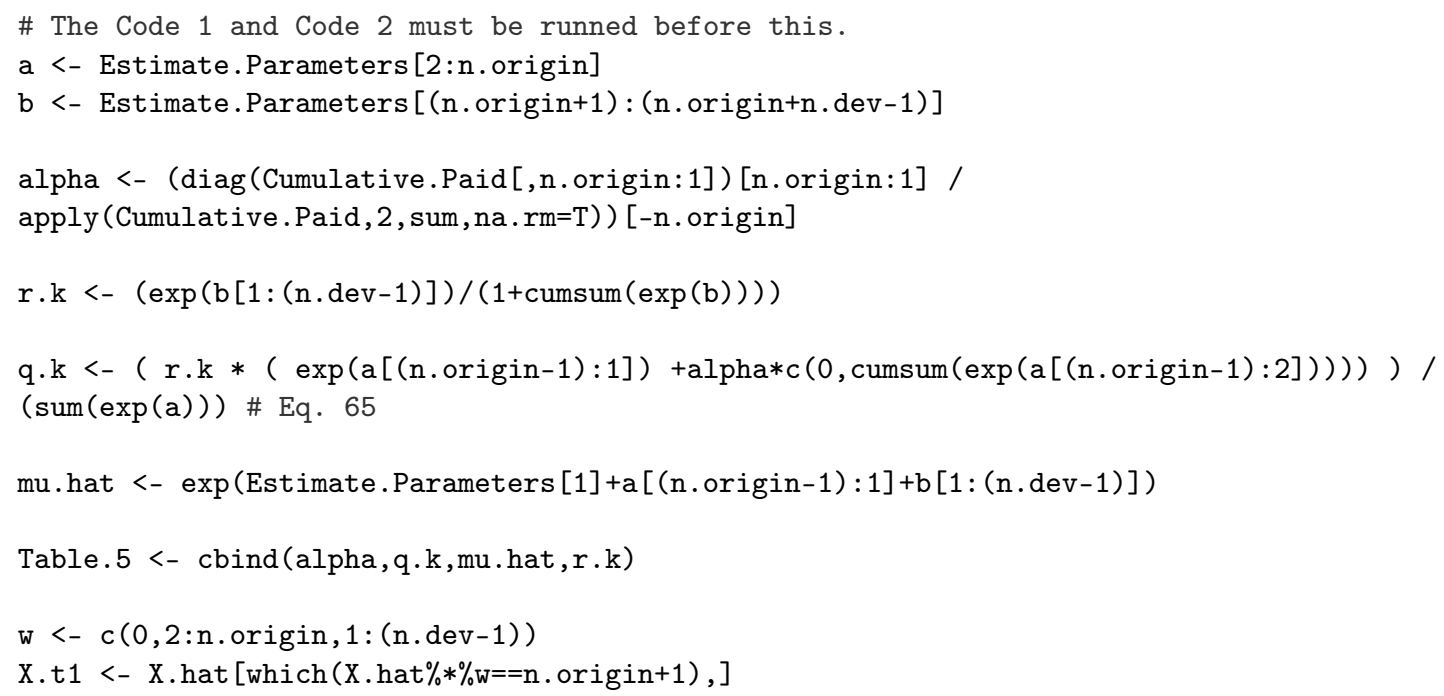


Listing 3. Cont.

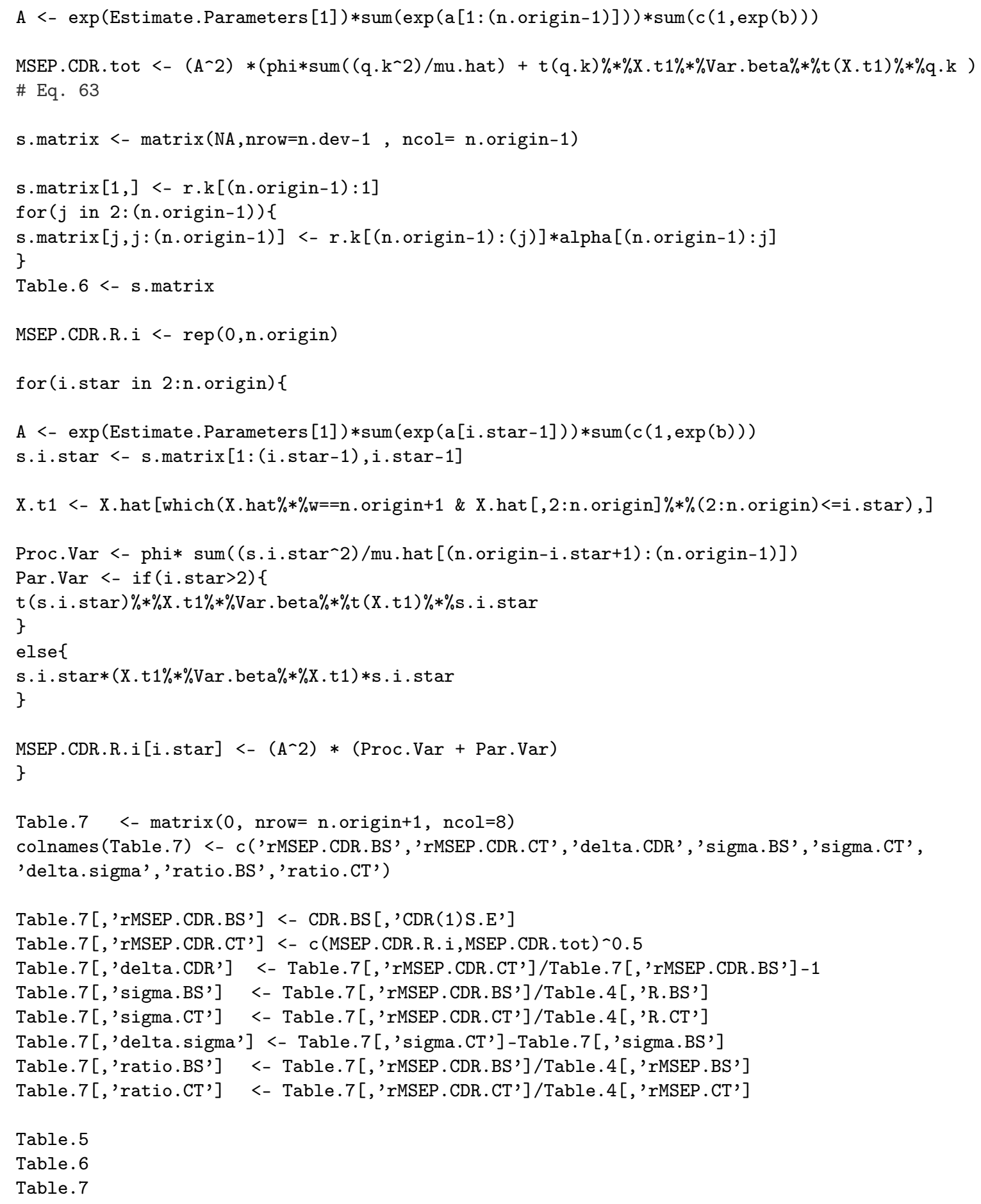

\section{Conclusions}

In this work, after a short review about the claims reserve volatility in the GLM frameworkcalculated in an ultimate view-we have drawn the formulae to calculate the one-year volatilities of the specific ODP model. Nonetheless, while the ultimate view is drawn on the basis of the incremental payments under an indipendence general assumption, the second one year volatility is derived through the use of the chain ladder cumulative payments estimate. The consequence is the one-year volatility 
of a specific accident year is calculated also according to the previous accident year estimates, similarly to the Merz-Wüthrich model.

Therefore, these formulae could generally be a valid alternative to the Merz-Wüthrich formulae regarding volatility parameters, or undertaking specific parameters (USP), in order to calculate the Solvency Capital Requirement according to the Solvency II framework. Furthermore, these results could also be used to solve the potential distortions caused by approaches such as, for instance, the emergence pattern introduced by England and linked to the single accident year (Casualty Actuaries of Europe Fall Meeting 2009, Zurich) . This approach has been mainly developed to overcome the cases where the bootstrapping is not appropriate, like, for example, due to triangle stability matters or computational reasons linked to the re-reserving.

To summarize, the emergence-pattern is based on the assumption that volatility at the ultimate level gradually emerges in time, so that, if a total volatility estimate can be determined, mechanisms that allow for making it emerge by using a specific pattern can also be created. In this case, we assume the Claims Development Result (CDR) to be a best estimate function; as a consequence, the CDR standard deviation is calculated through this relation, by estimating one factor for each of the observed accident years.

Basically, the calibration problem is dealt with by building the factors on the ratios between the CDR and the ultimate cost standard deviations for each accident year-followed by the proper equalizations to calibrate one pattern applicable to each accident year.

Indeed, the distortion can appear when the ultimate view volatilities are obtained by using the ODP - through a closed-tool or bootstrapping technique-but are then distributed in time by using factors drawn from the ratios between Merz-Wüthrich and Mack formulae, thus drawn from a different model. The results from this paper will make these approaches-typical of current practice in the internal models building-more coherent.

Author Contributions: These authors contributed equally to this work.

Funding: This research received no external funding.

Acknowledgments: We thank Riccardo Cesari, Milena Nocente and Valentino Pompili for helpful comments in a previous version of this paper. Stefano Cavastracci and Agostino Tripodi would like to remark that this article reflects the personal view of the authors and not necessarily that of IVASS.

Conflicts of Interest: The authors declare no conflict of interest.

\section{Abbreviations}

The following abbreviations are used in this manuscript:

$\begin{array}{ll}\text { BS } & \text { Bootstrap } \\ \text { CDR } & \text { Claims Develpoment Result } \\ \text { CT } & \text { Closed Tool } \\ \text { GLM } & \text { Generalized Linear Model } \\ \text { MSEP } & \text { Mean Square Error of Prediction } \\ \text { rMSEP } & \sqrt{M S E P} \\ \text { USP } & \text { Undertaking Specific Parameters }\end{array}$

\section{References}

Antonio, Katrien, and Jan Beirlant. 2008. Issues in claims reserving and credibility: A semiparametric approach with mixed models. Journal of Risk and Insurance 75: 643-76. [CrossRef]

Bjökwall, Susanna, Hössjer Ola, Ohlsson Esbjörn, and Richard Verrall. 2011. A generalized linear model with smoothing effects for claims reserving. Insurance: Mathematics and Economics 49: 27-37.

Despeyroux, Aurélie, Charles Levi, Christian Partrat, and Jerôme Vignancour. 2003. Techniques for valuation a general insurance company within the framework of IAS standards: Some proposals. Paper presented at the XXXIV International ASTIN Colloquium, Berlin, Germany, August 24-27. 
England, Peter D., and Richard J. Verrall. 2001. A flexible framework for stochastic claims reserving. Proceedings of the Casualty Actuarial Society 88: 1-38.

England, Peter D., and Richard J. Verrall. 2002. Stochastic claims reserving in general insurance. British Actuarial Journal 8: 443-544. [CrossRef]

Gigante, Patrizia, and Luciano Sigalotti. 2004. Valutazione della riserva sinistri con i GLM nel contesto dei nuovi standard contabili. In Quaderni del Dipartimento di Matematica Applicata alle Scienze Economiche Statistiche e Attuariali "B. de Finetti". Trieste: Universitá di Trieste, vol. 6.

Hudecovà, Sarka, and Michal Pešta. 2013. Modeling Dependencies in Claims Reserving with GEE. Insurance: Mathematics and Economics 53: 786-94. [CrossRef]

Leong, Weng Kah, Shaun S. Wang, and Han Chen. 2014. Back-Testing the ODP Bootstrap of the Paid Chain-Ladder Model with Actual Historical Claims Data. Variance 8: 182-202.

Merz, Michael, and Mario V. Wüthrich. 2015. Stochastic Claims Reserving Manual: Advances in Dynamic Modeling. SSRN Manuscript 2649057. Geneva: Swiss Finance Institute.

Nelder, J. A., and D. Pregibon. 1987. An extended quasi-likelihood function. Biometrika 74: 221-32. [CrossRef]

Renshaw, A. E., and Richard Verrall. 1998. A stochastic model underlying the chain-ladder technique. British Actuarial Journal 4: 903-23. [CrossRef]

Röhr, Ancus. 2016. Chain Ladder and Error Propagation. ASTIN Bulletin 46: 1-38.

Taylor, Greg C., and Gráinne McGuire. 2004. Loss Reserving with GLMs: A Case Study. Research Paper 113. Centre for Actuarial Studies, University of Melbourn, Australia. Sydney: Institute of Actuaries, pp. 489-99.

Taylor, Greg C., and Gráinne McGuire. 2016. Stochastic Loss Reserving Using Generalized Linear Models. CAS Monograph No. 3. Arlington: Casualty Actuarial Society.

Venter, Gary G. 2007. Generalized Linear Models beyond the Exponential Family with Loss Reserve Applications. ASTIN Bulletin 37: 345-64. [CrossRef]

Wedderburn, R. W. M. 1971. Quasi-likelihood functions, generalized linear models, and the Gauss-Newton method. Biometrika 61: 439-47.

(C) 2018 by the authors. Licensee MDPI, Basel, Switzerland. This article is an open access article distributed under the terms and conditions of the Creative Commons Attribution (CC BY) license (http:/ / creativecommons.org/licenses/by/4.0/). 